\title{
Floral and Leaf Anatomy of Hibiscus Species
}

\author{
U. A. Essiett ${ }^{*}$, E. S. Iwok \\ Department of Botany and Ecological Studies University of Uyo, P. M. B. 1017, Uyo. Akwa Ibom State-Nigeria \\ *Corresponding author: u.essiett@yahoo.com
}

Received September 27, 2014; Revised October 10, 2014; Accepted October 21, 2014

\begin{abstract}
Comparative anatomical studies of the leaves and flowers of $H$. arnottianus, $H$. surattensis, $H$. acetosella and $H$. rosa-sinensis are described. The anisocytic stomata was the commonest followed by brachyparacytic, anomocytic, staurocytic stomata and laterocytic stomatas respectively. H. acetosella are distinguished on other species by having laterocytic stomata on both surfaces of leaves and parallel contiguous stomata are found on abaxial surface while in $H$. rosa-sinensis laterocytic is found only on adaxial surface. There are five different types of abnormal stomata, unopened stomatal pore, two stomata sharing one subsidiary cell, parallel contiguous stomata and aborted guard cell found in all the surfaces of the leaves and flowers. In addition parallel contiguous stomata are found on adaxial surface of $H$. rosa-sinensis and abaxial surface of $H$. arnottianus flower. $H$. rosa-sinensis had five-armed trichome on the abaxial surface that helps in distinguishing it from other species studied. Crystal druses are only present on both adaxial surface of $H$. arnottianus and $H$. rosa-sinensis leaf and on the abaxial surface of $H$. acetosella flower. The shape of epidermal cells, anticlinal cell walls, guard cell areas, stomatal index and trichomes varied. The results obtained could be used as diagnostic tool for plant identification and preparation of monograph on the species.
\end{abstract}

Keywords: Hibiscus, Malvaceae, guard cell area, stomatal index, trichome, anticlinal cell walls

Cite This Article: U. A. Essiett, and E. S. Iwok, "Floral and Leaf Anatomy of Hibiscus Species." American Journal of Medical and Biological Research, vol. 2, no. 5 (2014): 101-117. doi: 10.12691/ajmbr-2-5-1.

\section{Introduction}

The family Malvaceae is one of the most important families consisting of 82 genera and 1,500 species with Hibiscus over 200 species, Sida 200 species, Abutilon 190 species and Malva 40 species. The family is world wide in distribution but is mostly represented in the tropical and subtropical region. Members may be herbs, shrub or the trees with mucilage. The plants Hibiscus rosa-sinensis, Hibiscus surattensis, Hibiscus arnottainus and Hibiscus acetosella belong to the sub-kingdom Tracheobionta (vascular plants). Division Magnoliopsida and family Malvaceae [1].

Hibiscus, rosa-sinensis, young leaves are sometimes used as a spinach substitute. Flowers of Hibiscus rosasinensis can also be made into a kind of pickle or used as a purple dye for colouring foods such as preserved fruit and cooked vegetables. Root is edible but very fibrousy. $H$. rosa-sinensis flower preparations are used for hair care. The flowers themselves are edible and are used in salads in the Pacific Islands. The flowers are used to shine shoes in parts of India. The red flowers of Hibiscus acetosella and possibly also the leaves are occasionally used to make a tea, somewhat similar to the use of the red calyces of Hibiscus sabdariffa. The root of $H$. acetosella is edible but insipid and fibrous. Pink - or red flowers are often grown as ornamental plants in gardens. Some people in Cameroon and Democratic Republic of Congo combine the use of Hibiscus acetosella as a vegetable with its use as a hedge to separate plots. A red leaved cultivar of $H$. acetosella is also grown for its attractive foliage [2].

Hibiscus sabdariffa is said to lower fevers and high blood pressure, relieve coughs, increase urination and kill bacteria. China rose ( $H$. rosa-sinensis) is used primarily for respiratory problems but also for skin disorders and to treat fevers. Hibiscus syriacus (rose of sharon) is used externally as an emollient to soften and soothe the skin as well as used internally for digestive disorder [3].

The essential oil in the seeds of $H$. rosa-sinensis has a strong antispasmodic effect and has been successfully used to ease the pain for intestine pile or kidney colic. The flower extract is used internally in the treatment of excessive and painful menstruation. Veneral diseases and to promote hair growth [4] Hibiscus rosa-sinensis extract exerted potential protective effect against tumor. Promotion stage of cancer development [5] and the anthocyanins extract of this plant grown in Egypt proved pronounced cytotoxic activities against hepatoma and breast cancer cell lines [6].

The leaves of $H$. rosa-sinensis are emollient, aperients, anodyne and laxative, leaves and stembark are used for abortion. The staminal column is diuretic used for kidney trouble. The flowers are astringent, demulcent, emollient, refrigerant, constipating, hypoglyucaemic, aphrodisiac, emmenagogue and used for treating aloparia, burning sensation in the body, diabetes and menstrual disorders. The buds are used for treatment of vaginal and uterine discharges. Leaves and flowers are good for healing ulcers and for promoting growth and colour of hair. The leaves 
and flowers of $H$. rosa-sinensis are also beaten into a paste and poulticed onto cancerous swellings and mumps.

In Angola, an infusion of the leaves of Hibiscus acetosella in water is used as post-fever tonic, it is also used as medicine to treat anaemia. In East Africa, children with an aching body are washed in cold water to which some mashed Hibiscus acetosella leaves have been added [2].

In recent times, the classification of Hibiscus species in Nigeria still pose some problems due to variation within taxa and high degree of hybridization. Previous pharmacological investigation of the genus Hibiscus indicated the presence of some species with useful biological activities as antihypertensive, antiinflammatory, anti-pyretic, hepatoprotective, antidiarrhoeic, anti-spematogenic , anti-tumour, anti-diabetic, anti- convulsivant, anti-helminthic immunodulator, antioxidant and antimutagenic agents [7-15].

Presence of various types of glandular and eglandular trichomes is a characteristics features of genus Hibiscus. Scientific interest in plants trichome is based on their functional and taxonomic importance and on the economic usefulness of some trichome-generated products. Trichome may serve a variety of defensive and physiological functions. Leaf trichomes have been shown to reduce insect herbivory in a number of plant species $[16,17,18]$.

For plants in xeric habitats, epidermal appendages reflect light and can reduce transpiration rate $[19,20]$. The micromorphological characteristics of foliar trichomes have played an important role in plant systematic especially of particular groups at generic and specific levels. Such type of studies in the field has fascinated plant morphologists and systematists towards the diversity of trichomes features [21].

Spring [22] stated that the presence or absence of peltate hairs and their form, size and colour could be used in distinguishing between genera and species, but also their corresponding parts, thus being important in pharmacognosy, archaeobotany , paleobotany and agronomy [23].

Adedeyi and Illoh [24] reported the comparative foliar anatomy of the genus Hibiscus, but they concentrated on the general anatomy and added only few sentences about foliar trichomes without any morphological descriptions and anatomical measurements. In their work, they found out that the eight major types of glandular and eglandular foliar trichomes, have been observed.

Nwachukwu and Mbagwu [25] reported on anatomical features of the roots and leaves of Hibiscus rosa-sinensis and Abelmoschus esculenta results obtained from it showed presence of long chain and numerous epidermal cells in Hibiscus rosa-sinensis while they are short chains small and numerous epidermal cells in Abelmoschus esculenta. Priya et al. [26] pointed out the stomatal variation in cultivars of Hibiscus rosa-sinensis L. Calcium oxalate crystals may form in any organ or tissue within plants, crystals occur in roots, stems, leaves, flowers, fruits and seeds [27] and within epidermal , ground and vascular tissues [28].

The study was to ascertain the taxonomic importance of flowers and leaf anatomical features in deducing similarities and differences among the taxa studied and utilizing the anatomical characters obtained from these taxa for the systematic grouping and characterization of the taxa. The study attempted to reveal additional characteristics for Hibiscus species which might be useful for identification of the species studied at the generic and species level.

\section{Materials and Methods}

\subsection{Plant Collection}

The fresh leaves and flowers of Hibiscus rosa-sinensis, $H$. arnottianus, $H$. surattensis and $H$. acetosella were collected in September 2012 in Uyo Local Government Area of Akwa Ibom State. The plants were authenticated by Dr. (Mrs.) U. A. Essiett, a taxonomist in the Department of Botany and Ecological studies. Anatomical studies were carried out using the methods below.

\subsection{Microscopic Examination}

For the purpose of anatomical studies small sizeable portions were obtained from standard median part of the leaves of matured and well expanded leaves. Epidermal peels of both abaxial and adaxial surfaces were made by placing the leaf blade taken from standard median portion of the leaves on clean glass slab. The specimen surfaces to be studied was placed facing down; it was irrigated with water holding down the specimen from one end. The epidermis above the desired surface was scrapped off carefully with a sharp razor blade. Loose cells were washed away from the epidermal peel with the aid of soft carmel hairbrush and watered until the desired epidermis below was reached. The epidermis peels were stained in $1 \%$ aqueous solution of Safranin 0 for 4 to 8 minutes rinsed carefully in water to remove excess stain and then mounted in $10 \%$ glycerol. Guard cells area was calculated by Francos constant method (Guard cells area = length $\mathrm{x}$ width $x$ 0.7854). The stomatal index was determined according to Metcalfe and Chalk (1979) using the formula:

$$
\frac{S}{E+S} \times \frac{100}{1}=\text { Stomatal Index }(S I)
$$

Where: $\mathrm{S}=$ number of stomata per unit area

$\mathrm{E}=$ number of epidermal cells in the same area

All measurements were made with the aid of an Ocular micrometer and finally converted by the Ocular constant with respect to the power under which they were taken. Images were computerized digitally with a motic images plus version $2.0 \mathrm{ml}$ mounted on Zeiss light microscope.

\section{Results}

\subsection{H. arnottianus (M. Roe) D. Bates}

The leaf epidermal cell of the adaxial surface are rectangular to polygonal to irregular in shape while abaxial is polygonal to irregular. Adaxial cells (40 x 26 $\mu \mathrm{m})$ are smaller in size than that of the abaxial (56 x 23 $\mu \mathrm{m})$ as shown in Table 1.

Anticlinal cell walls of adaxial surface are straight to slightly undulating while abaxial surface are straight to undulating. Peltate trichome, bifurcated (two-armed) trichome, unicellular nonglandular trichome are found on 
the adaxial surface while peltate trichome, four-armed sessile stellate trichome , unicellular trichome, two-armed trichome are found on abaxial surface (Plate 1: B, C, D, K, $\mathrm{M}, \mathrm{N}$ and $\mathrm{O}$, Table 3)

Stomata distribution in the investigated taxa is hypoamphistomatic (stomata abundant on abaxial surface and scanty on adaxial surface).

The mature stomata are mostly anisocytic, anomocytic, staurocytic and brachyparacytic (Plate 1: A, F. H, I, J, P, S and $\mathrm{T}$ ) found on both surfaces which are distributed and their axes are oriented in different directions. Staurocytic stomata is abundant. Guard cell area on adaxial surface (5 $\mu \mathrm{m})$ is higher than those of abaxial surface $(4 \mu \mathrm{m})$. Abnormal stomata with unopened stomatal pore and stomata sharing one subsidiary cell are present on abaxial surface but aborted guard cells are found on both surfaces (Plate 1: G, L, Q, R). Crystal druses are common on adaxial surfaces (Plate $1 \mathrm{E}$ ).

The flower anticlinal cell wall of the adaxial surface are slightly undulate while abaxial surface are sinous or undulate (Table 2). The flower epidermal cell on adaxial are polygonal to irregular while abaxial is polygonal.

Stomatas are scanty on the adaxial surface and abundant on the abaxial surface (hypoamphistomatic). The mature stomata types were anisocytic, anomocytic and staurocytic on the abaxial surfaces while on the adaxial surface staurocytic stomata are mostly frequent followed by anisocytic (Plate 8: B, C, D, E, F).

The lowest guard cells were recorded in adaxial surface (5 $\mu \mathrm{m})$ and the highest was found on abaxial surface (7 $\mu \mathrm{m}$, Table 2). Abnormal stomatas are parallel contiguous stomata on the abaxial surfaces only (Plate $8 \mathrm{H}$ ). Glandular trichome are found on both surfaces (Plate 8: A, G, Tab. 3). Crystal druses were absent.

\subsection{H. surattensis $L$.}

Epidermal cells on the adaxial surface of the leaf are pentagonal to polygonal while abaxial surface are polygonal to irregular. Abaxial cells $(65 \times 30 \mu \mathrm{m})$ are smaller in size than adaxial cells $(78 \times 28 \mu \mathrm{m})$ as shown in Table 1. Anticlinal cell walls of adaxial surface are undulate while abaxial cells are straight to sinous. Curved trichome on vein cells, two-armed non-glandular trichome on vein cells, glandular trichome on the abaxial surface while on the adaxial surface are glandular trichome (Plate 2: C, D, K, P,U, Table 3). Stomatal distribution in the investigated taxa is hypoamphistomatic (stomatas abundant on abaxial and scanty on adaxial surface).

The mature stomatas are mostly anisocytic, brachyparacytic, laterocytic, staurocytic on both surface but in addition, anomocytic stomatas were present on the abaxial surface only (Plate 2: A, B, H, I, L, M, N, S, T). Guard cell area in adaxial surface $(7 \mu \mathrm{m})$ was smaller than that of abaxial surface ( $8 \mu \mathrm{m}$, Tab. 1$)$. Abnormal stomata with aborted guard cells, one guard cell and unopened stomatal pore were observed on both surfaces but only 2 stomata sharing one subsidiary cell were found on abaxial surface (Plate 2: E, F, G, J, O, Q, R) .

The flower epidermis of the adaxial surface are polygonal to irregular while abaxial surface are pentagonal to polygonal to irregular. The anticlinal cell walls of the adaxial epidermis are straight undulating while abaxial are undulating (Table 2). Stomatas are completely absent on both abaxial and adaxial surfaces (Table 2). Glandular trichomes are present on only the abaxial surface (Plate 7B, Table 3). Crystal druses are absent in all the surfaces.

\subsection{H. acetosella Welw. Ex Hiern.}

Epidermal cells on the adaxial surface of the leaf are pentagonal to polygonal to irregular while abaxial surface are polygonal to irregular. Abaxial cells (59 x $24 \mu \mathrm{m})$ are smaller than adaxial cells $(75 \times 34 \mu \mathrm{m})$ as shown in Tab. 1 . Anticlinal cell walls of adaxial surface are slightly undulating while abaxial surface is undulating. Few glandular trichomes are found on the abaxial surface (Plate 3F). Stomatal distribution in the investigated taxa is amphistomatic (stomatas are abundantly present on both surfaces). Mature stomatas found on abaxial surface are anisocytic, staurocytic, and laterocytic while anisocytic, brachyparacytic, few anomocytic and laterocytic stomatas are found on the adaxial surface and anisocytic are more abundant (Plate 3: A, B, J, K, M). Guard cell area in abaxial $(5 \mu \mathrm{m})$ was smaller than that of adaxial $(14 \mu \mathrm{m}$, Table 1). Abnormal stomata with aborted guard cell, one guard cell and unopened stomatal pore were observed on both surfaces while parallel contiguous stomata and two stomata sharing one subsidiary cell were found only on the abaxial surface. (Plate 3; C, D, E, G, H, I, L, N). Crystals were completely absent.

The flower epidermis of the adaxial surface are polygonal to irregular while the abaxial are polygonal to irregular. The anticlinal cell walls of the adaxial are slightly undulating while abaxial are undulating. Stomatas are scanty on abaxial surface and completely absent on adaxial surface (hypostomatic Table 2). Anisocytic stomata is abundant. Mature stomatas are Anomocytic, staurocytic, for adaxial surface only. The guard cell area was recorded in abaxial surface only (5 $\mu \mathrm{m}$, Table 2) while adaxial was absent. Abnormal stomata with aborted guard cell was observed (Plate 6E). Glandular trichomes are present on both surfaces but peltate trichome was found on adaxial surface only (Plate 6: B, F, N, Tab. 3). Crystals were common on both surfaces (Plate 6: D, G).

\subsection{H. rosa-sinensis $L$.}

Epidermal cells on the abaxial surface are pentagonal to polygonal while adaxial cells are pentagonal to polygonal to irregular with cuticular striations. Abaxial cells (65 x 39 $\mu \mathrm{m})$ are smaller than adaxial cells $(70 \times 34 \mu \mathrm{m})$ as shown in Table 1. Anticlinal cell walls of abaxial surface are straight to curved, slightly undulate, while adaxial are slightly undulate. Trichomes on abaxial surface were glandular, two-armed trichome, four-armed, three-armed and five -armed non-glandular trichome on vein cells and only glandular on the adaxial surface (Table 3, Plate 4: E, H, J, K, L, M, R). Stomata distribution in the investigated taxa is amphistomatic (stomatas are abundant on both surfaces). Mature stomatas are anisocytic, staurocytic, anomocytic and brachyparacytic on both surfaces, while laterocytic stomatas were found on the adaxial surface only (Plate 4: A, B, C, F, N, P, Q, S). Guard cell area in adaxial $(9 \mu \mathrm{m})$ is smaller than that of abaxial $(12 \mu \mathrm{m}$, Tab. 1). Abnormal stomata with parallel contiguous stomata was found in adaxial surfaces but aborted guard cell and 
unopened stomatal pore on abaxial surface (Plate $4 \mathrm{~B}, \mathrm{D}$, G, I, J, T).

The flower epidermis of the adaxial surface are polygonal toirregular while that of abaxial surface are polygonal to irregular. The anticlinal cell walls of abaxial surfaceis sinous to undulate while adaxial surface is slightly undulate. Stomatas are scanty on adaxial surface and abundant on the abaxial surface (hypoamphistomatic). Anisocytic, anomocytic and staurocytic are found on both surfaces. Staurocytic are abundant on both surfaces. (Plate
5F, G, J, L, M, O, P). The lowest guard cell was recorded in abaxial surfaces $(8 \mu \mathrm{m})$ while adaxial had $(9 \mu \mathrm{m}$, Tab. 2). Abnormal stomata with parallel contiguous stomata and one guard cell were found on the adaxial surface but only aborted guard cell were present on abaxial surface of the flower. (Plate $5 \mathrm{H}, \mathrm{L}, \mathrm{N}$ ). Three-armed, two-armed, unicellular, non-glandular and glandular trichomes were present. Non- glandular on abaxial surfaces but glandular on abaxial surface only (Plates 5 A, B, C, D, E, Table 3). Crystal druses are absent on both surfaces.

Table 1. Epidermal feature of the leaves of Hibiscus arnottianus, $\mathrm{H}$. surattensis, $\mathrm{H}$. rosa-sinensis and $\mathrm{H}$. acetosella

\begin{tabular}{|c|c|c|c|c|c|c|c|c|c|c|c|}
\hline \multirow[t]{2}{*}{ Species } & \multicolumn{2}{|c|}{$\begin{array}{l}\text { Stomatal size } \\
(\mu \mathrm{m})\end{array}$} & \multicolumn{2}{|c|}{$\begin{array}{c}\text { Epidermal cells } \\
\qquad(\mu \mathrm{m})\end{array}$} & \multicolumn{2}{|c|}{$\begin{array}{l}\text { Guard Cells } \\
(\mu \mathrm{m})\end{array}$} & \multicolumn{2}{|c|}{$\begin{array}{l}\text { Stomatal } \\
\text { Index (\%) }\end{array}$} & \multirow[t]{2}{*}{$\begin{array}{c}\text { Stomatal } \\
\text { Distribution }\end{array}$} & \multicolumn{2}{|c|}{ Epidermal Cell wall } \\
\hline & Ad & $\mathrm{Ab}$ & Ad & $\mathrm{Ab}$ & Ad & $\mathrm{Ab}$ & Ad & $\mathrm{Ab}$ & & Ad & $\mathrm{Ab}$ \\
\hline & $\mathrm{LxB}$ & $\mathrm{LxB}$ & LxB & LxB & & & & & & & \\
\hline $\begin{array}{c}\text { Hibiscus } \\
\text { arnottianus }\end{array}$ & $30 \times 20$ & $35 \times 20$ & $40 \times 26$ & $56 \times 23$ & 5 & 4 & 7 & 43 & hypoamphistomatic & $\begin{array}{l}\text { Straight to } \\
\text { Slightly undulate }\end{array}$ & $\begin{array}{l}\text { Straight to } \\
\text { undulate }\end{array}$ \\
\hline H. surattensis & $26 \times 10$ & $28 \times 14$ & $78 \times 28$ & $65 \times 30$ & 7 & 8 & 11 & 34 & hypoamphistomatic & undulate & $\begin{array}{l}\text { Straight to } \\
\text { sinous }\end{array}$ \\
\hline H. acetosella & $32 \times 18$ & 29x15 & $75 \times 34$ & $59 \times 24$ & 14 & 5 & 22 & 38 & amphistomatic & Slightly undulate & undulate \\
\hline $\begin{array}{l}\text { H. rosa- } \\
\text { sinensis }\end{array}$ & $26 \times 17$ & $34 \times 19$ & $70 \times 34$ & $65 \times 39$ & 9 & 12 & 44 & 39 & amphistomatic & Slightly undulate & $\begin{array}{l}\text { Slightly } \\
\text { undulate }\end{array}$ \\
\hline
\end{tabular}

Table 2. Epidermal feature of the flowers of Hibiscus arnottianus, $\mathrm{H}$. surattensis, $\boldsymbol{H}$. rosa-sinensis and $\mathrm{H}$. acetosella

\begin{tabular}{|c|c|c|c|c|c|c|c|c|c|c|c|}
\hline \multirow[t]{2}{*}{ Species } & \multicolumn{2}{|c|}{$\begin{array}{l}\text { Stomatal size } \\
(\mu \mathrm{m})\end{array}$} & \multicolumn{2}{|c|}{$\begin{array}{l}\text { Epidermal cells } \\
(\mu \mathrm{m})\end{array}$} & \multicolumn{2}{|c|}{$\begin{array}{l}\text { Guard Cells } \\
(\mu \mathrm{m})\end{array}$} & \multicolumn{2}{|c|}{$\begin{array}{c}\text { Stomatal } \\
\text { Index (\%) }\end{array}$} & \multirow[t]{2}{*}{$\begin{array}{c}\text { Stomatal } \\
\text { Distribution }\end{array}$} & \multicolumn{2}{|c|}{ Epidermal Cell wall } \\
\hline & Ad & $\mathrm{Ab}$ & Ad & $\mathrm{Ab}$ & Ad & $\mathrm{Ab}$ & Ad & $\mathrm{Ab}$ & & Ad & $\mathrm{Ab}$ \\
\hline & LxB & LxB & $\mathrm{LxB}$ & LxB & & & & & & & \\
\hline $\begin{array}{c}\text { Hibiscus } \\
\text { arnottianus }\end{array}$ & $39 \times 26$ & $32 \times 22$ & $88 \times 17$ & $76 \times 26$ & 5 & 7 & 13 & 4 & amphistomatic & $\begin{array}{c}\text { Slightly } \\
\text { undulate }\end{array}$ & $\begin{array}{l}\text { Sinous or } \\
\text { undulate }\end{array}$ \\
\hline H. surattensis & - & - & $44 \times 18$ & $75 \times 42$ & - & - & - & - & - & $\begin{array}{l}\text { Straight to } \\
\text { undulate }\end{array}$ & $\begin{array}{l}\text { Slightly } \\
\text { undulate }\end{array}$ \\
\hline H. acetosella & - & $27 \times 20$ & $35 \times 25$ & $57 \times 20$ & - & 5 & - & 25 & amphistomatic & $\begin{array}{c}\text { Slightly } \\
\text { undulate }\end{array}$ & undulate \\
\hline $\begin{array}{l}\text { H. rosa- } \\
\text { sinensis }\end{array}$ & $26 \times 15$ & $27 \times 16$ & $36 \times 16$ & $65 \times 29$ & 9 & 8 & 25 & 4 & hypoamphistomatic & $\begin{array}{l}\text { Slightly } \\
\text { undulate }\end{array}$ & $\begin{array}{c}\text { Undulate or } \\
\text { Sinous }\end{array}$ \\
\hline
\end{tabular}

Table 3. Trichomes characteristic of leaves and flowers of Hibiscus arnottianus, $\mathrm{H}$. surattensis, $\mathrm{H}$. rosa-sinensis and $\boldsymbol{H}$. acetosella

\begin{tabular}{|c|c|c|c|c|c|c|}
\hline Species & $\begin{array}{c}\text { Glandular } \\
\text { trichomes } \\
\text { Leaves } \\
\text { Ad Ab } \\
\end{array}$ & $\begin{array}{c}\begin{array}{c}\text { Non-glandular } \\
\text { trichomes }\end{array} \\
\mathrm{Ad} \mathrm{Ab} \\
\end{array}$ & $\begin{array}{c}\text { Peltate } \\
\text { Trichomes } \\
\text { Ad Ab } \\
\end{array}$ & $\begin{array}{c}\text { Glandular } \\
\text { Trichomes } \\
\text { Flowers } \\
\text { Ad Ab } \\
\end{array}$ & $\begin{array}{c}\text { Non-glandular } \\
\text { Trichomes } \\
\text { Ad Ab } \\
\end{array}$ & $\begin{array}{c}\text { Peltate } \\
\text { Trichomes } \\
\text { Ad Ab } \\
\end{array}$ \\
\hline & LxB LxB & LxB LxB & LxB LxB & LxB LxB & LxB LxB & LxB LxB \\
\hline $\begin{array}{c}\text { Hibiscus } \\
\text { arnottianus }\end{array}$ & -- & 170x29 277x64 & $257 \times 42$ 244x38 & 261x38 252x42 & -- & -- \\
\hline H. surattensis & $237 \times 42$ 262x62 & $289 \times 40$ 181x38 & -- & $-262 \times 32$ & - & - \\
\hline H. acetosella & $-286 \times 42$ & -- & - & $257 \times 39276 x 48$ & -- & - 362x38 \\
\hline H. rosa-sinensis & $316 x 44$ 197x42 & $253 \times 40$ & - & $-147 x 43$ & 278x62 279x38 & - \\
\hline
\end{tabular}

Key: Absent, Ad: Adaxial Surface (upper), Ab: Abaxial Surface (lower), L: Length, B: Breadth Plate 1A - 1T.

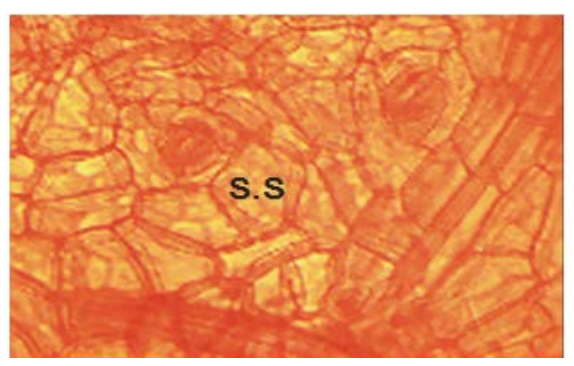

Plate 1A. A.S, Anisocytic and SS, staurocytic stomata of H. arnottianus (upper Surface) x 400

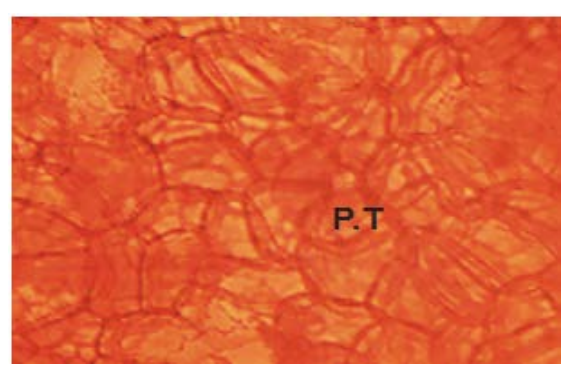

Plate 1B. P.T. Peltate trichome of H. arnottianus (upper Surface) x 400 


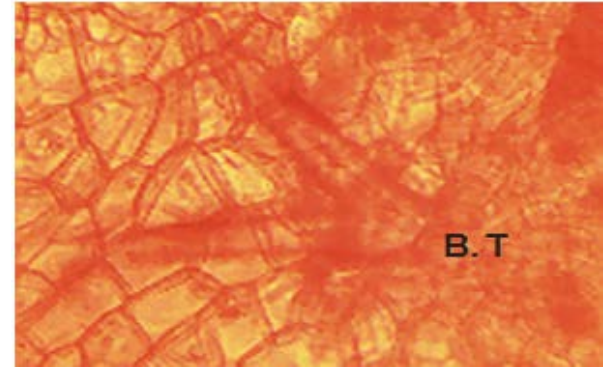

Plate 1C. B.T, Bifurcated (two-armed) trichome of $H$. arnottianus (upper Surface) x 400

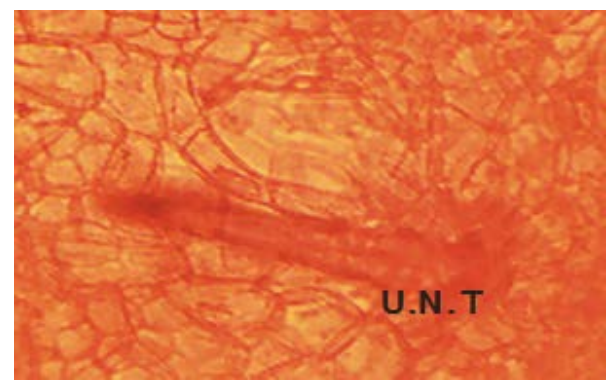

Plate 1D. U.N.T, Unicellular trichome of H. arnottianus (upper Surface) $\mathrm{x} 400$

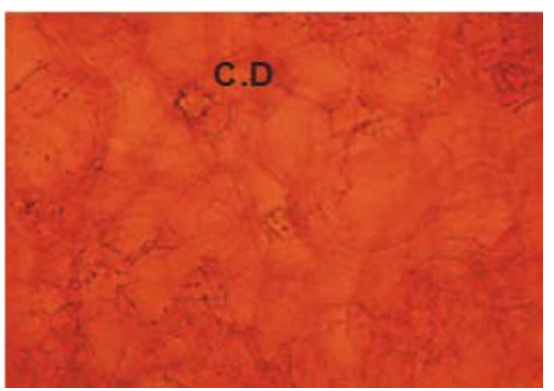

Plate 1E. C.D, Crystal druses of H. arnottianus (upper Surface) x 400

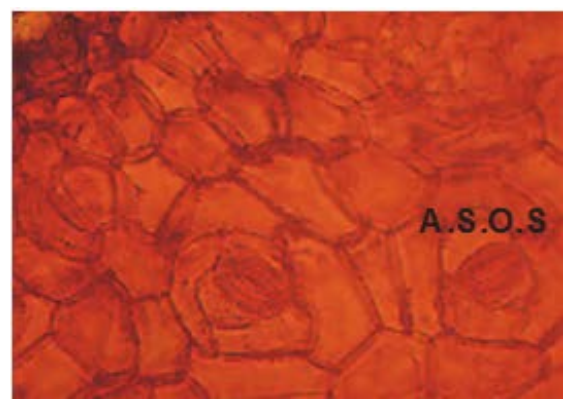

Plate 1F. A.S.O.S, Anomocytic stomata of $H$. arnottianus (upper Surface) $\mathrm{x} 400$

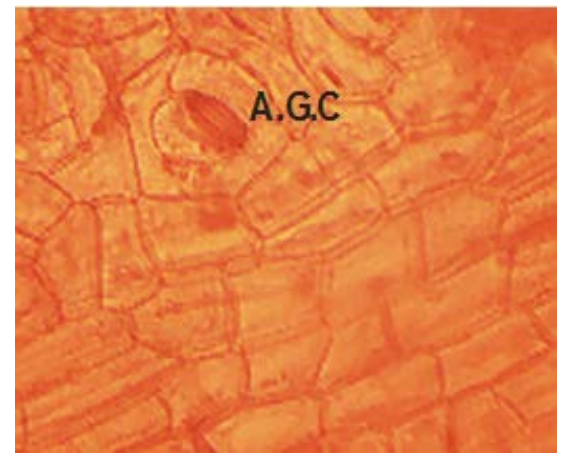

Plate 1G. A.GC, Aborted guard cell of H. arnottianus (upper Surface) x 400

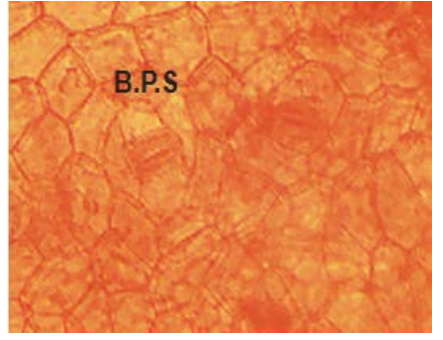

Plate 1H. B.P.S, Brachyparacytic of $H$. arnottianus (upper Surface) x 400

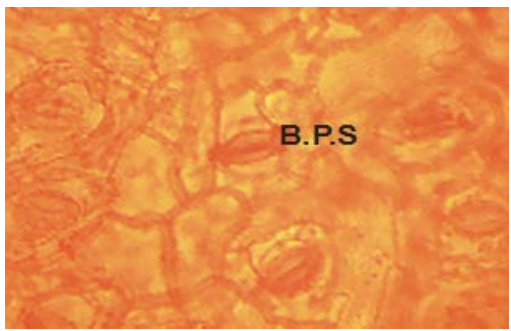

Plate 1I. B.P.S, Brachyparacytic of H. arnottianus (lower Surface) x 400

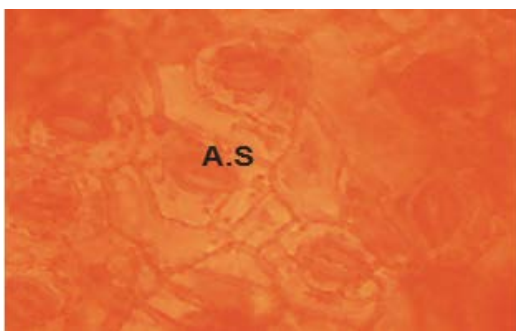

Plate 1J. A.S, Anisocytic of H. arnottianus (lower Surface) x 400

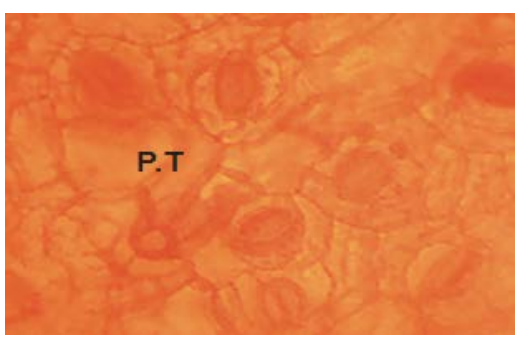

Plate 1K. P.T, Peltate of H. arnottianus (lower Surface) x 400

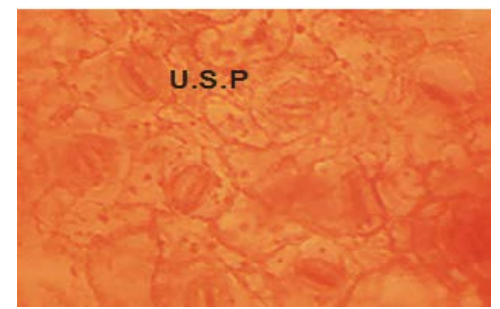

Plate 1L. U.S.T, Unopen stomatal pore of H. arnottianus (lower Surface) x 400

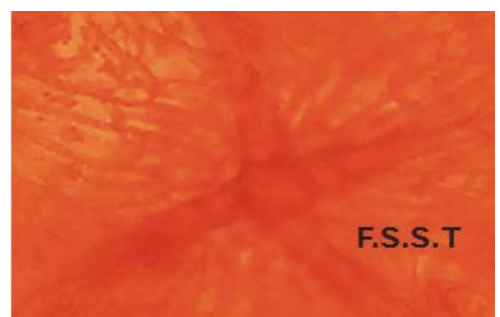

Plate 1M. U.S.T, Unopen stomatal pore of $H$. arnottianus (lower Surface) x 400 


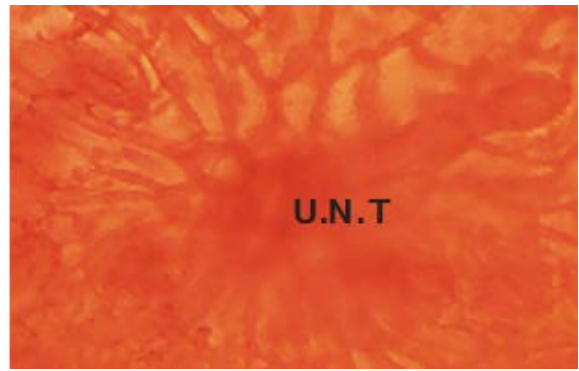

Plate 1N. U.N.T, Unicellular trichnome of H. arnottianus (lower Surface) $\mathrm{x} 400$

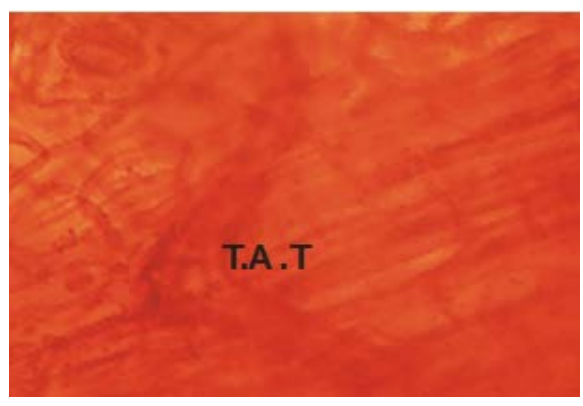

Plate 10. T.A.T, Two-armed (bifur cated) trichnome of $H$. arnottianus (lower Surface) x 400

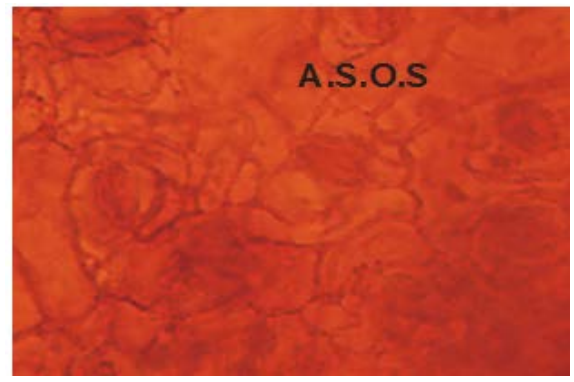

Plate 1P. A.O,A.S, Anomocytic stomata of $H$. arnottianus (lower Surface) $\mathrm{x} 400$

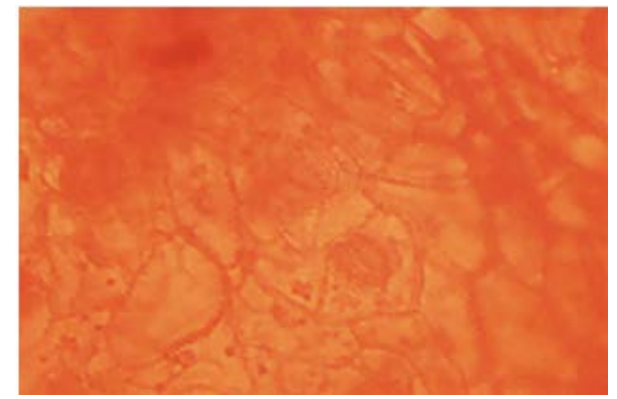

Plate 1Q. Two stomata sharing one subsidiary cell of $H$. arnottianus (lower Surface) x 400

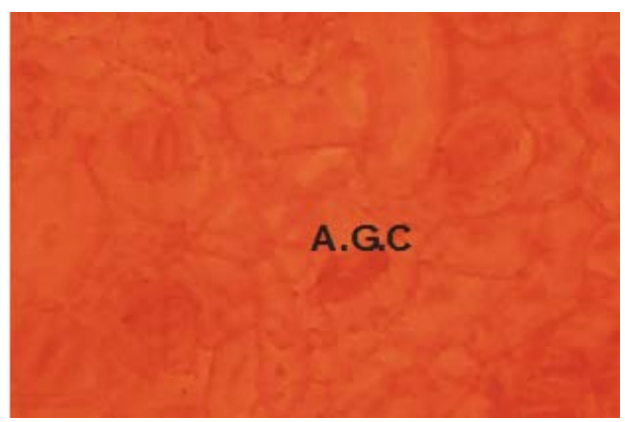

Plate 1R. A.G.C, Aborted guard cell of $H$. arnottianus (lower Surface) x 400

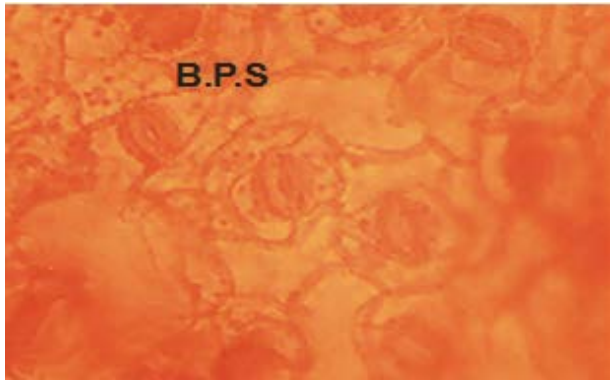

Plate 1S. B.P.S, Brachyparacytic stomata of $H$. arnottianus (lower Surface) $\mathrm{x} 400$

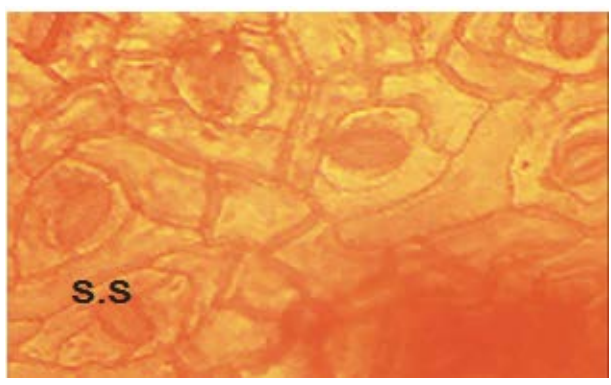

Plate 1T. S.S, Staurocytic stomata of $H$. arnottianus (lower Surface) $\mathrm{x}$ 400

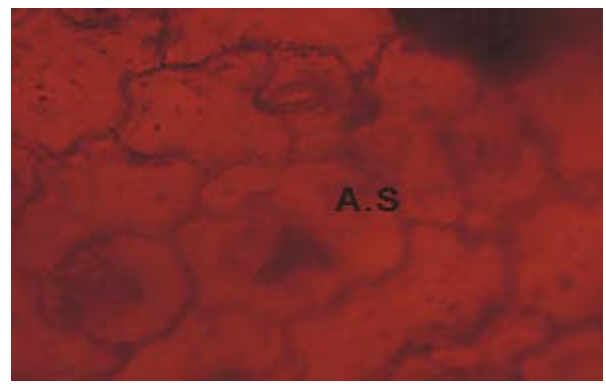

Plate 2A. A.S, Anisocytic stomata of $H$. surattensis (lower Surface) $\mathrm{x}$ 400

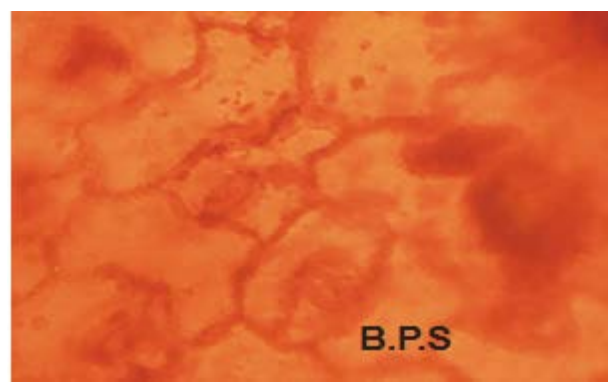

Plate 2B. B.P.S, Brachyparacytic stomata of $H$. surattensis (lower Surface) $\mathrm{x} 400$

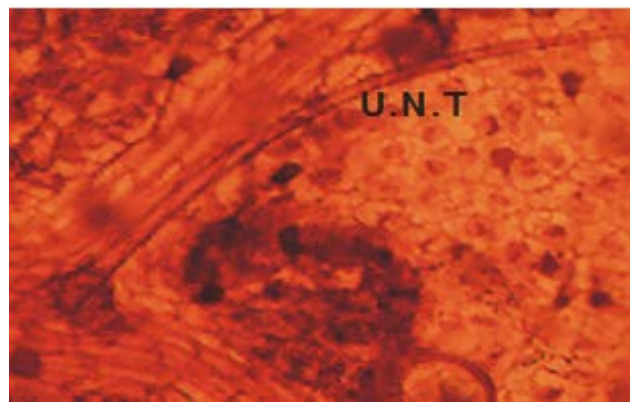

Plate 2C. U.N.T, Curved unicellular trichome of $H$. surattensis (lower Surface) x 100 


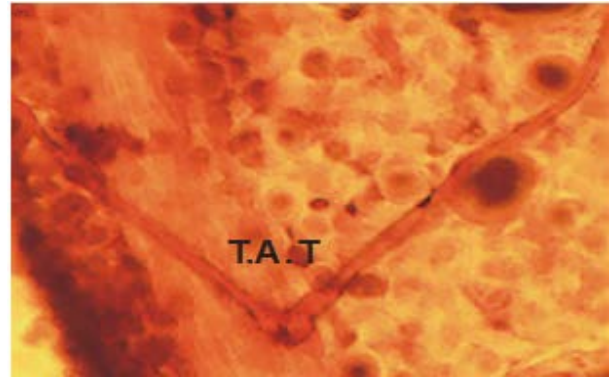

Plate 2D. T.A.T, Two-armed trichome of $H$. surattensis (lower Surface) $\mathrm{x} 100$

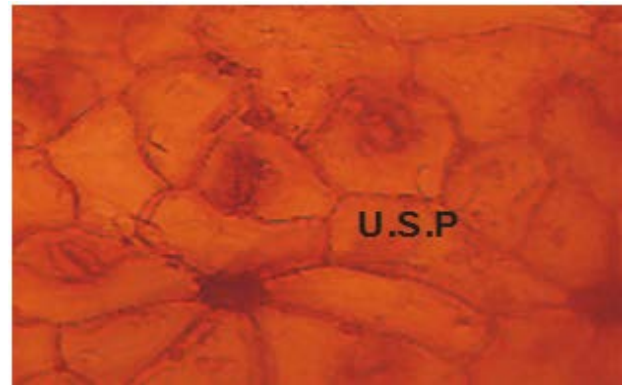

Plate 2E. U.P.S, Unopened stomatal pore of $H$. surattensis (lower Surface) x 400

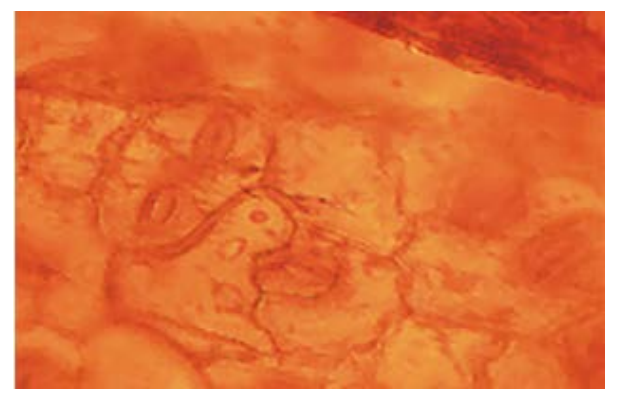

Plate 2F. Two stomata sharing one subsidiary cell of $H$. surattensis (lower Surface) x 400

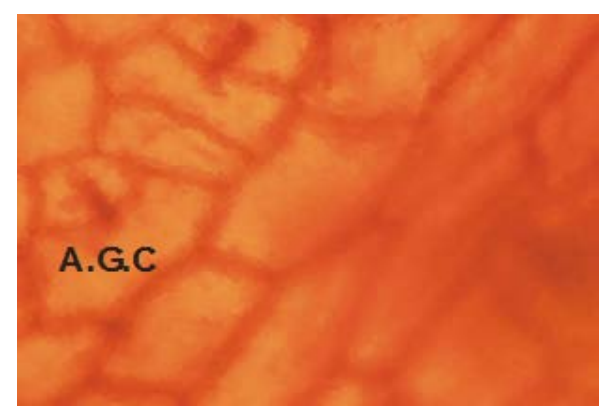

Plate 2G. A.G.C, Aborted guard cell of H. surattensis (lower Surface) x 400

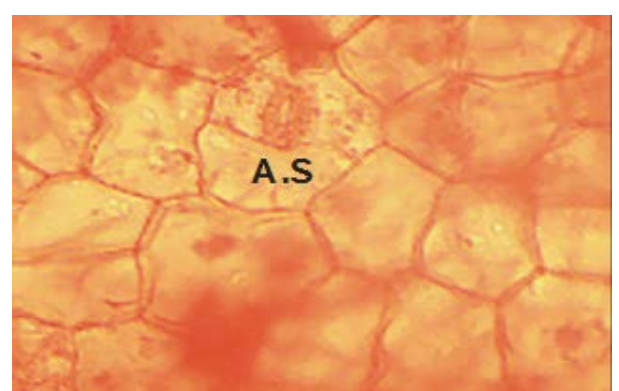

Plate 2H. A.S, Anisocytic stomata of $H$. surattensis (lower Surface) $\mathrm{x}$ 400

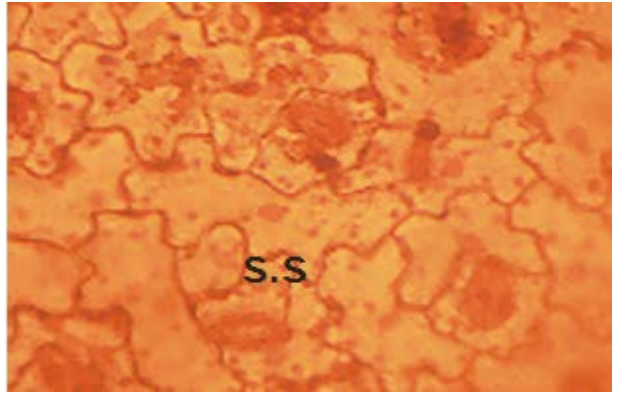

Plate 2I. S.S, Staurocytic stomata of $H$. surattensis (lower Surface) $\mathrm{x}$ 400

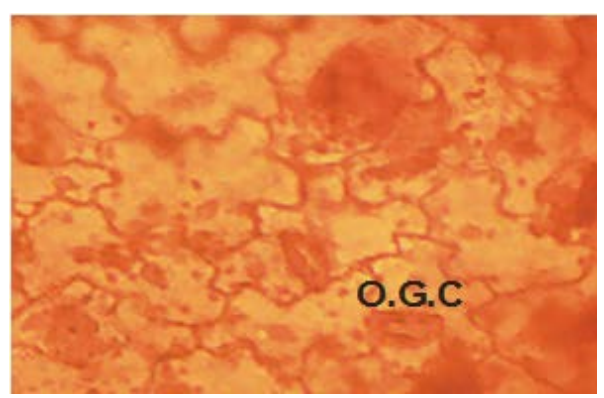

Plate 2J. O.G.C, One guard cell and two stomata sharing one subsidiary cell of $H$. surattensis (lower Surface) x 400

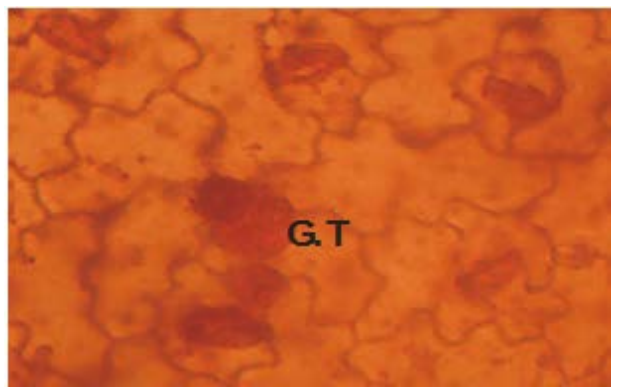

Plate 2K. G.T, Glandular trichome of $H$. surattensis (lower Surface) x 400

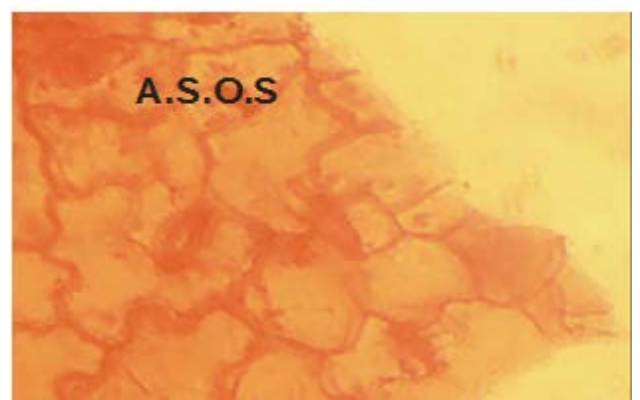

Plate 2L. A.S.O.S, Anomocytic stomata of H. surattensis (lower Surface) $\mathrm{x} 400$

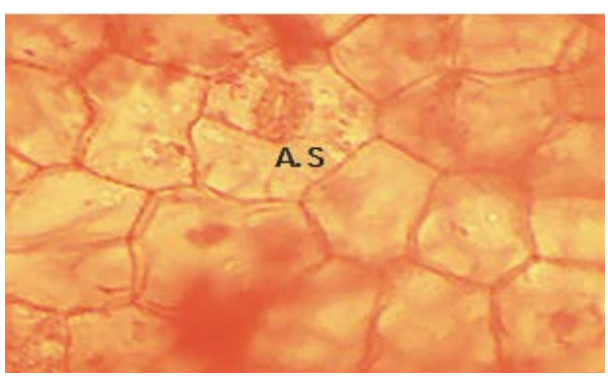

Plate 2M. A.S, Anisocytic stomata of $H$. surattensis (Upper Surface) x 400 


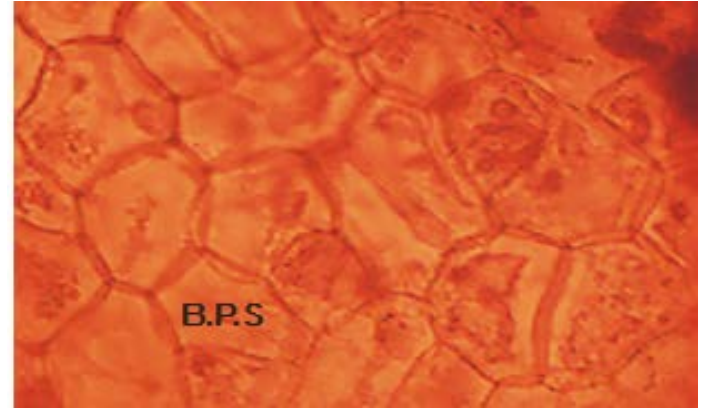

Plate 2N. B.P.S, Brachyparacytic stomata of $H$. surattensis (Upper Surface) $\mathrm{x} 400$

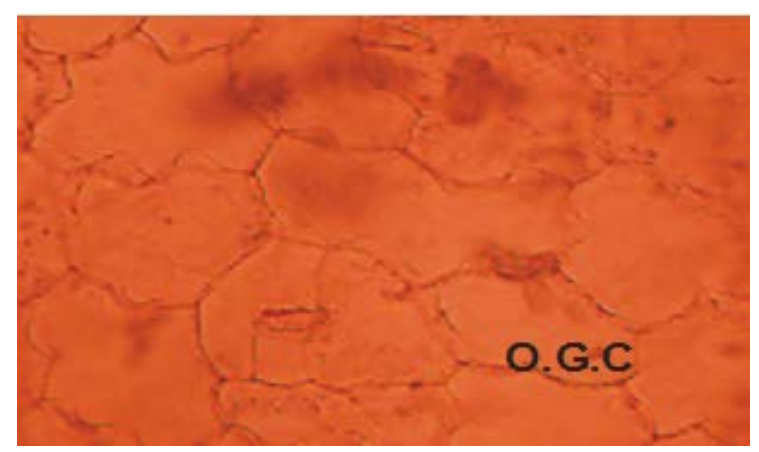

Plate 2O. O.G.C, One guard cell of H. surattensis (Upper Surface) x 400

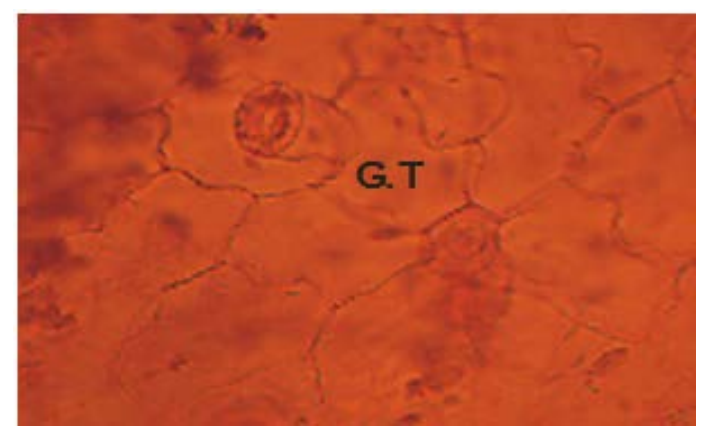

Plate 2P. G.T, Glandular trichome of $H$. surattensis (Upper Surface) $\mathrm{x}$ 400

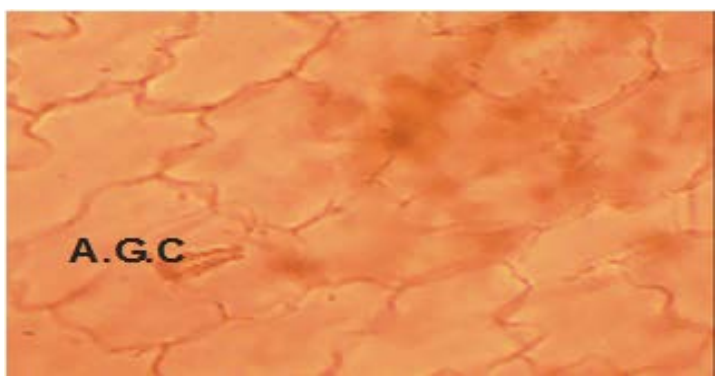

Plate 2Q. A.G.C, Aborted guard of H. surattensis (Upper Surface) x 400

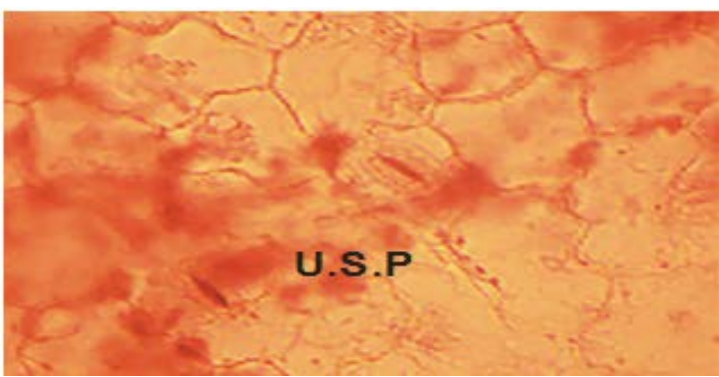

Plate 2R. U.S.P, Unopened stomatal pore of $H$. surattensis (Upper Surface) $\mathrm{x} 400$

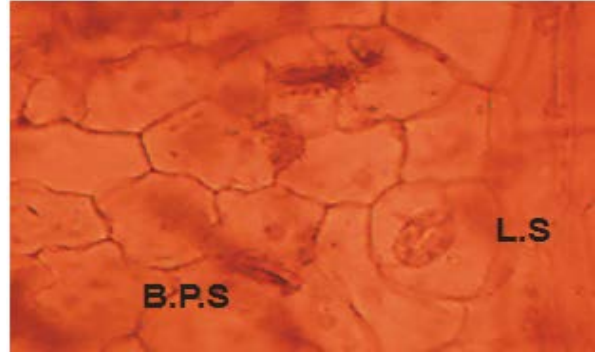

Plate 2S. B.P.S, Brachyparacytic and L.S, laterocytic stomata of $H$. surattensis (Upper Surface) x 400

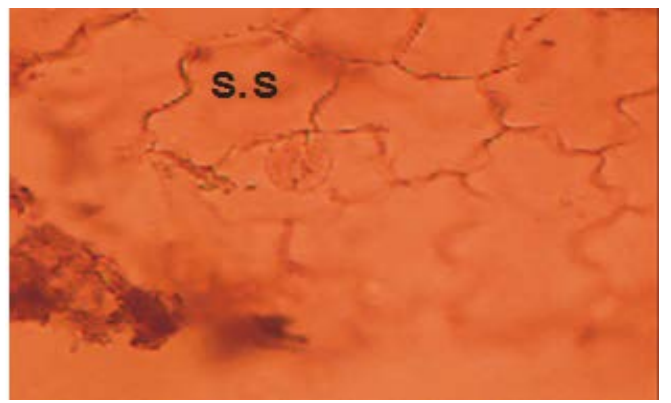

Plate 2T. S.S, Staurocytic stomata of $H$. surattensis (Upper Surface) $\mathrm{x}$ 400

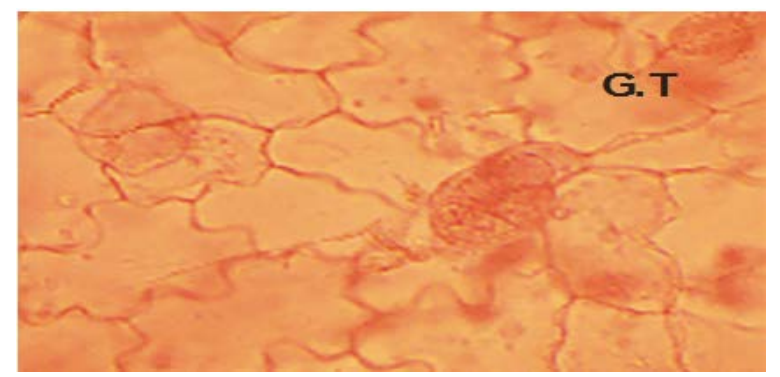

Plate 2U. G.T, Glandular trichome of $H$. surattensis (Upper Surface) x 400

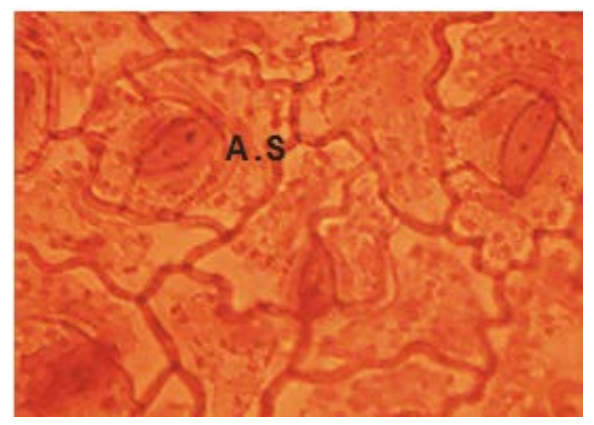

Plate 3A. A.S, Anisocytic stomata of $H$. acetosella (Lower Surface) $\mathrm{x}$ 400

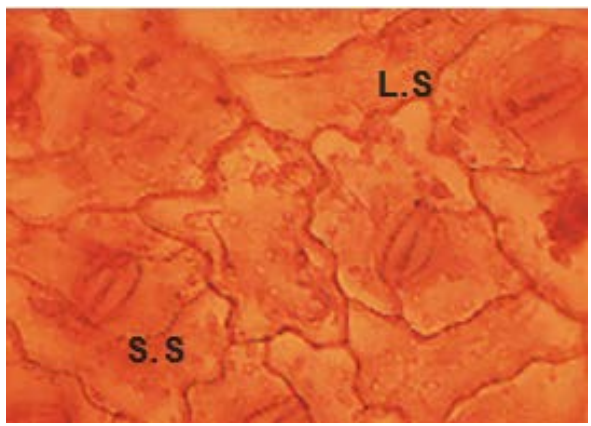

Plate 3B. L.S, Laterocytic stomata and S.S, staurocytic stomata of $H$. acetosella (Lower Surface) x 400 


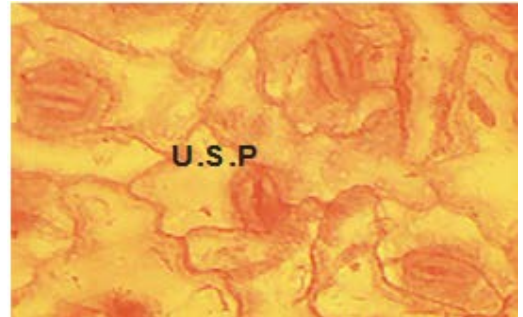

Plate 3C. U.S.P, Unopened stomata pore of $H$. acetosella (Lower Surface) x 400

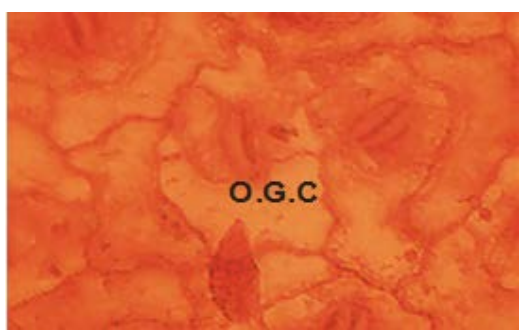

Plate 3D. O.G.C, One guard cell of H. acetosella (Lower Surface) x 400

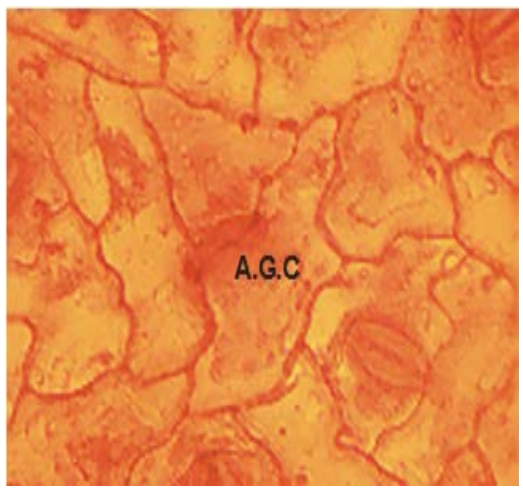

Plate 3E. A.G.C, Aborted sotomata of H. acetosella (Lower Surface) x 400

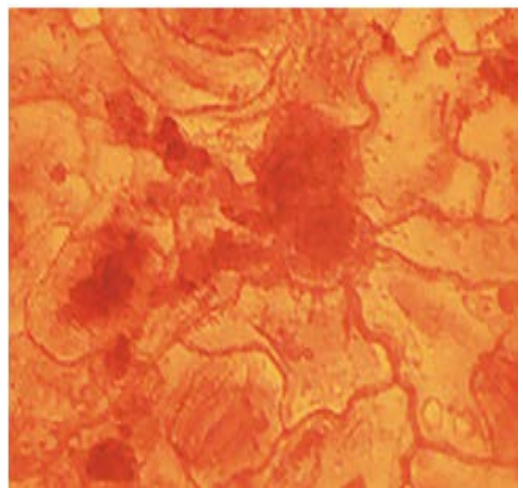

Plate 3F. G.T, Glandular trichome of $H$. acetosella (Lower Surface) x 400

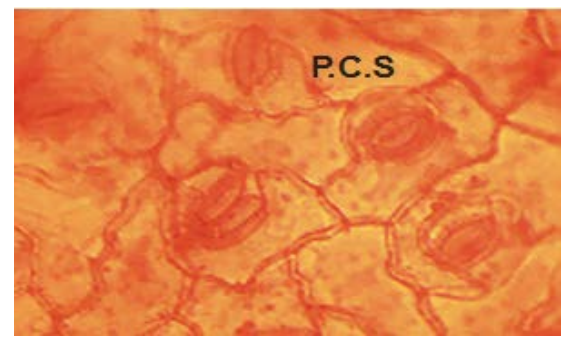

Plate 3G. P.C.S, Parallal contiguous stomata of $H$. acetosella (Lower Surface) $\mathrm{x} 400$

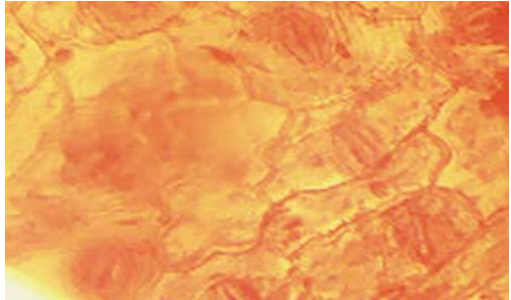

Plate 3H. Two stomata sharing one subsidiary cell of $H$. acetosella (Lower Surface) x 400

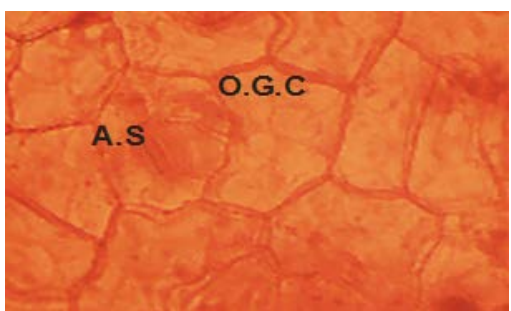

Plate 3I. O.G.C, One guard cell and A.S, anisocytic stomata of $H$. acetosella (upper Surface) x 400

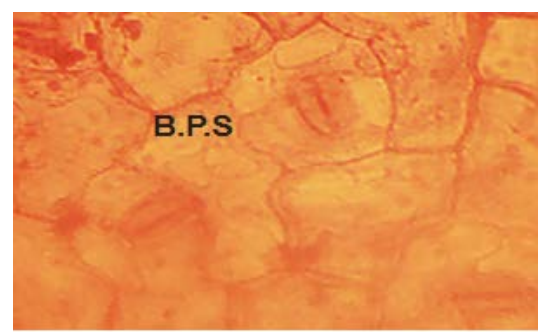

Plate 3J. B.P.S, Brachyparacytic stomata of H. acetosella (upper Surface) x 400

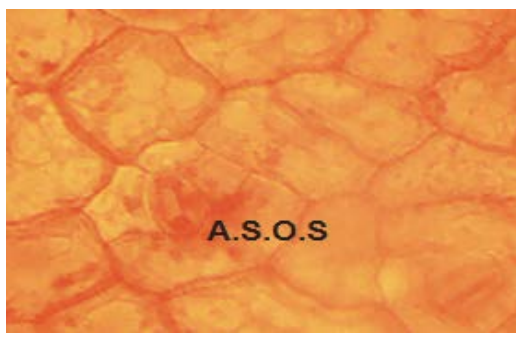

Plate 3K. A.S.O.S, Anomocytic stomata of H. acetosella (upper Surface) x 400

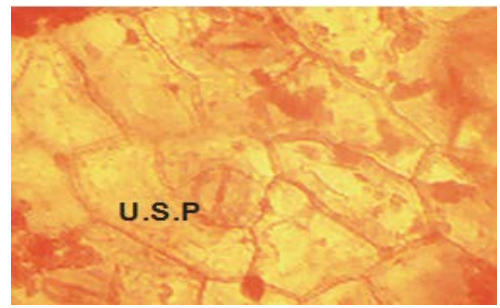

Plate 3L. U.S.P, Unopened stomata pore of $H$. acetosella (upper Surface) x 400

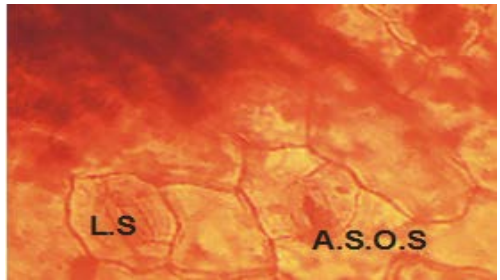

Plate 3M. L.S, Laterocytic stomata and A.S.O.S, Anomocytic stomata of H. acetosella (upper Surface) x 400 


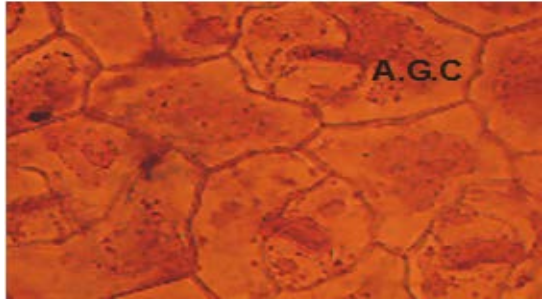

Plate 3N. A.G.C, Aborted guard cell of H. acetosella (upper Surface) x 400

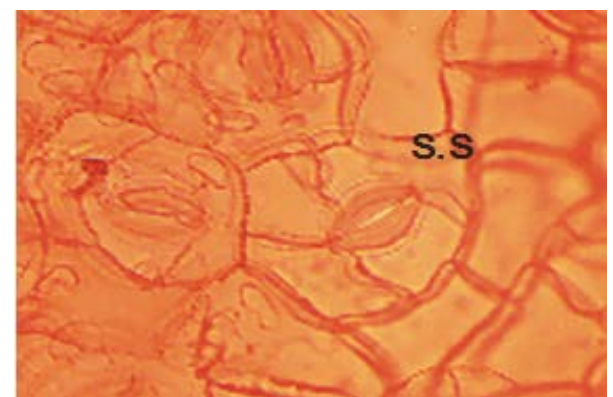

Plate 4A. S.S, Staurocytic stomata of $H$. rosa-sinensis (lower Surface) $\mathrm{x}$ 400

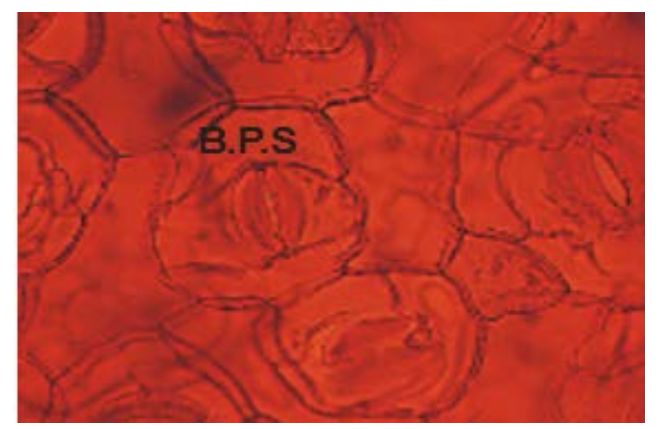

Plate 4B. B.P.S, Brachyparacytic stomata and one guard cell of $H$. rosasinensis (lower Surface) x 400

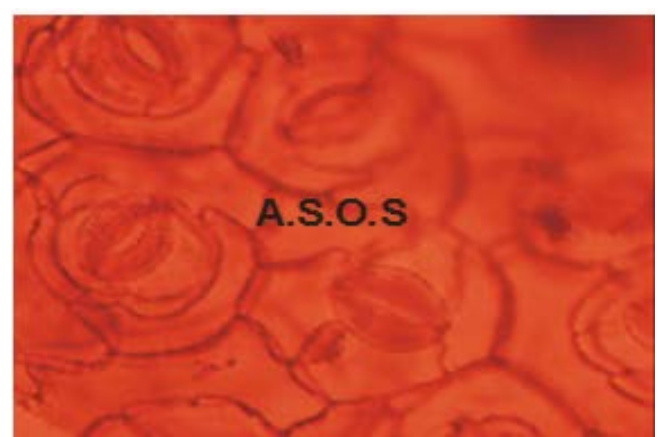

Plate 4C. A.S.O.S, Anomocytic stomata of H. rosa-sinensis (lower Surface) x 400

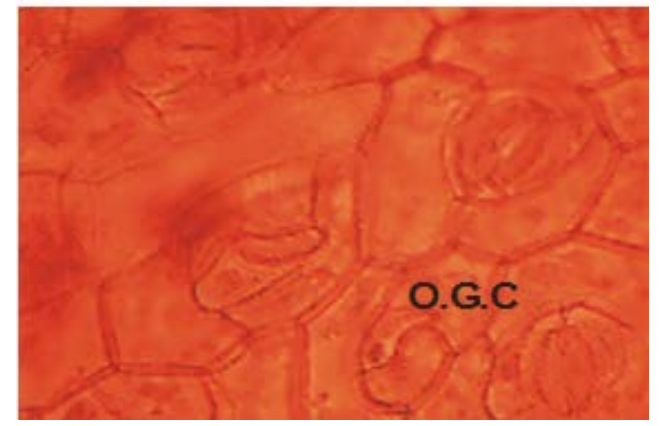

Plate 4D. O.G.C, One guard cell of H. rosa-sinensis (lower Surface) $\mathrm{x}$ 400

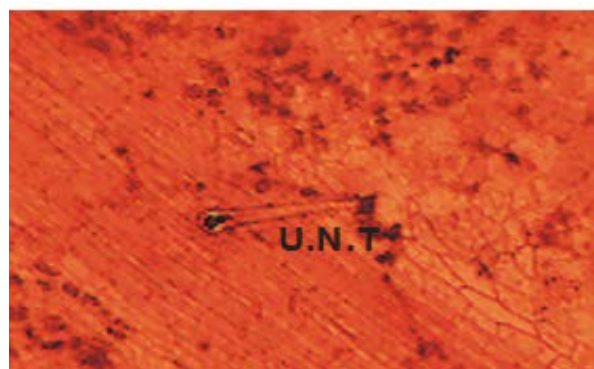

Plate 4E. U.N.T, Unicellular trichome on vein cells of $H$. rosa-sinensis (lower Surface) x 400

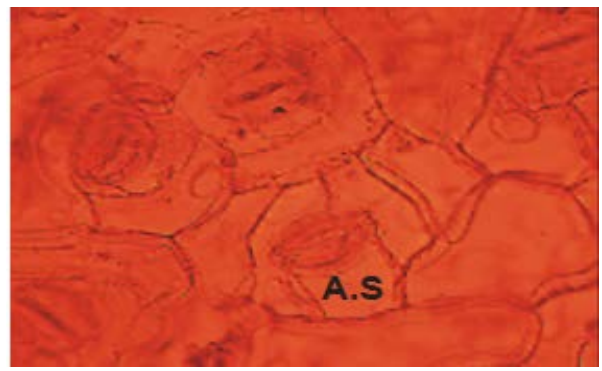

Plate 4F. A.S, Anisocytic stomata of $H$. rosa-sinensis (lower Surface) $\mathrm{x}$ 400

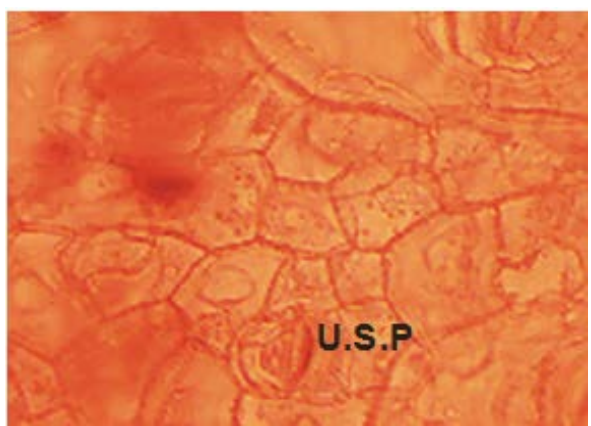

Plate 4G. U.S.P, Unopened stomata of H. rosa-sinensis (lower Surface) $\mathrm{x} 400$

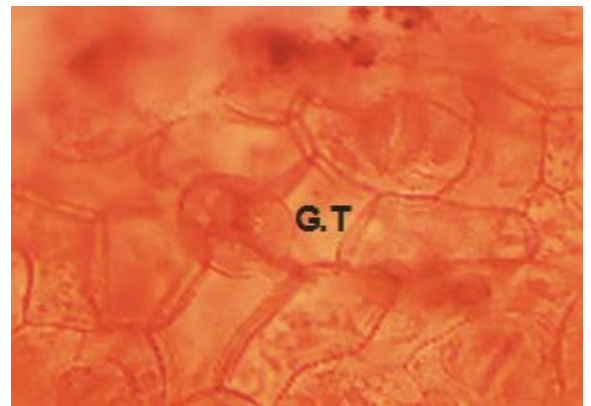

Plate 4H. G.T, Glandular trichome of H. rosa-sinensis (lower Surface) x 400

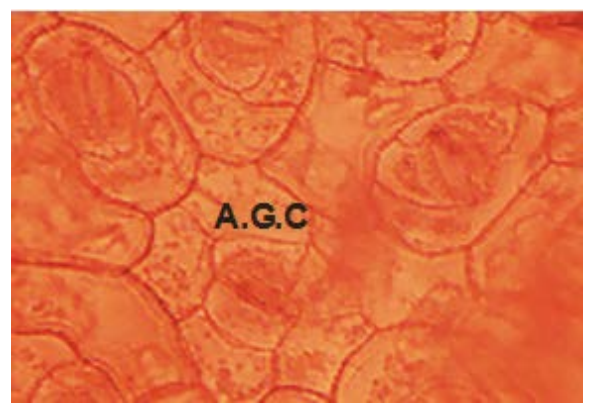

Plate 4I. A.G.C, Aborted stomata of H. rosa-sinensis (lower Surface) x 400 


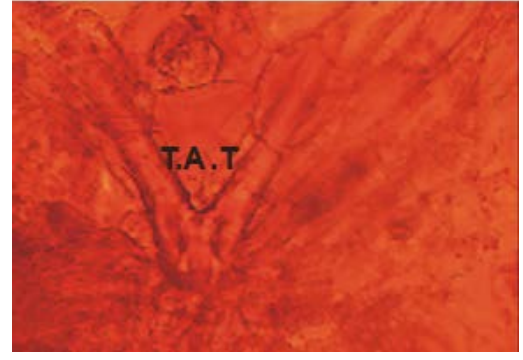

Plate 4J. T.A.T, Two-armed trichome of H. rosa-sinensis (lower Surface) $\mathrm{x} 400$

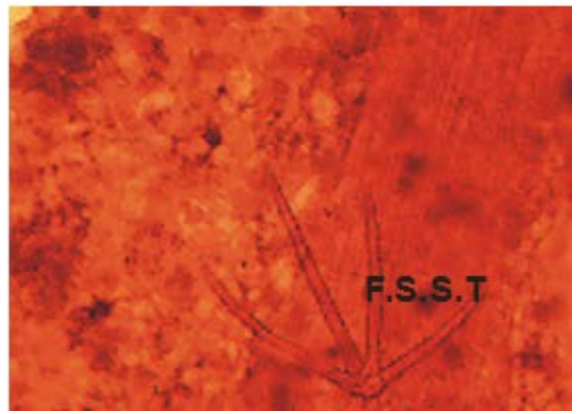

Plate 4K. F.S.S.T, Four-armed sessile stellate trichome of $H$. rosasinensis (lower Surface) x 100

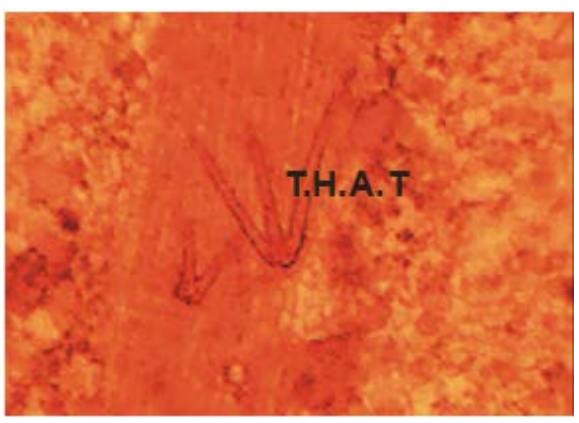

Plate 4L. T.H.A.T, Four-armed sessile stellate trichome of $H$. rosasinensis (lower Surface) x 400

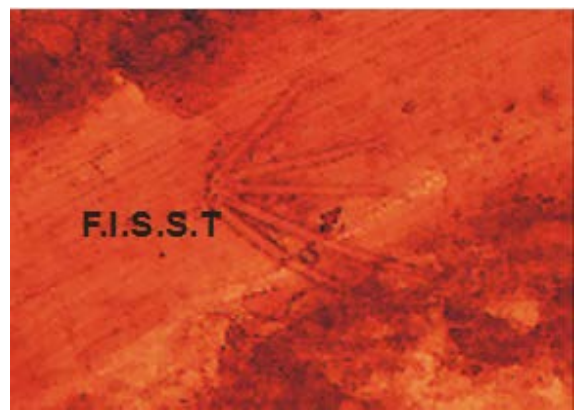

Plate 4M. F.I.S.S.T, FIVE-armed sessile stellate trichome of $H$. rosasinensis (lower Surface) x 100

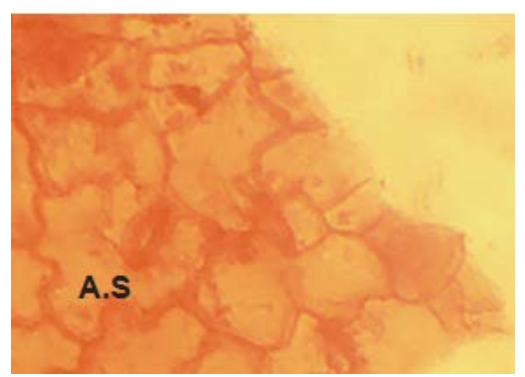

Plate 4N. A.S, Anisocytic stomata of $H$. rosa-sinensis (Upper Surface) x 400

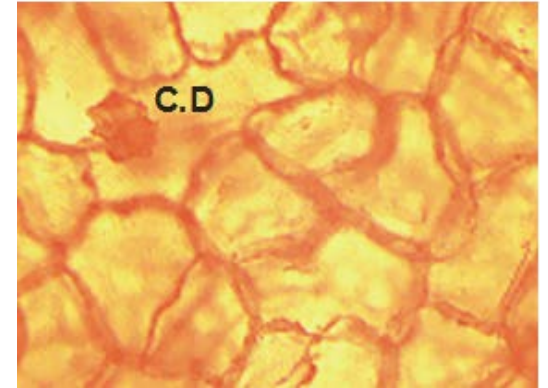

Plate 40. C.D, Crystal druses of H. rosa-sinensis (Upper Surface) x 400

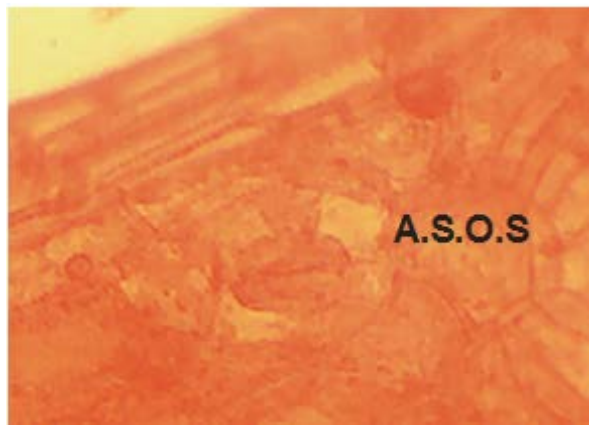

Plate 4P. A.S.O.S, Anomocytic stomata of H. rosa-sinensis (Upper Surface) x 400

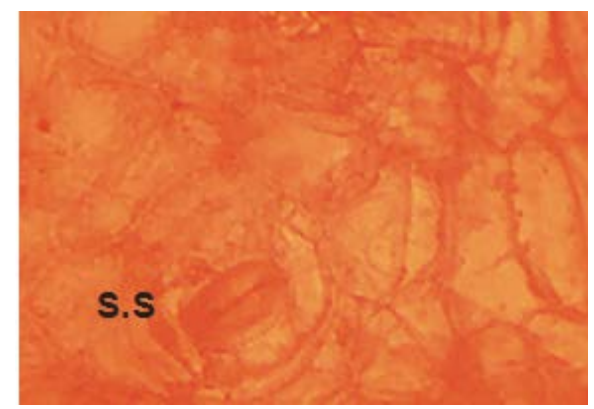

Plate 4Q. S.S, Staurocytic stomata of H. rosa-sinensis (Upper Surface) $\mathrm{x}$ 400

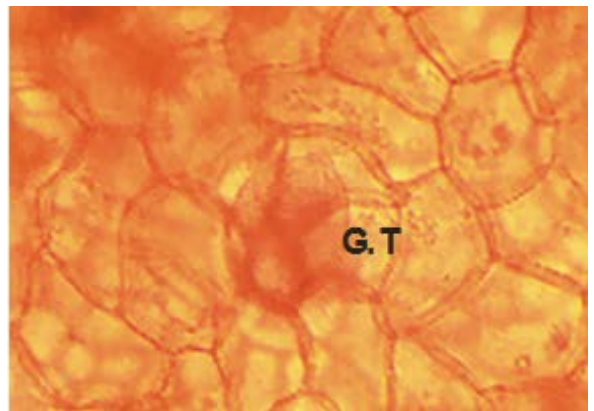

Plate 4R. G.T, Glandular trichome of $H$. rosa-sinensis (Upper Surface) $\mathrm{x}$ 400

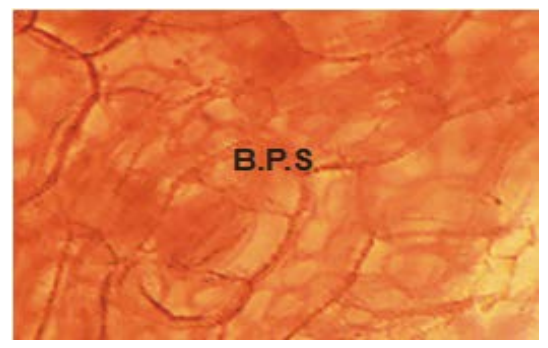

Plate 4S. B.P.S, Brach yparacytic stomata of $H$. rosa-sinensis (Upper Surface) $\mathrm{x} 400$ 


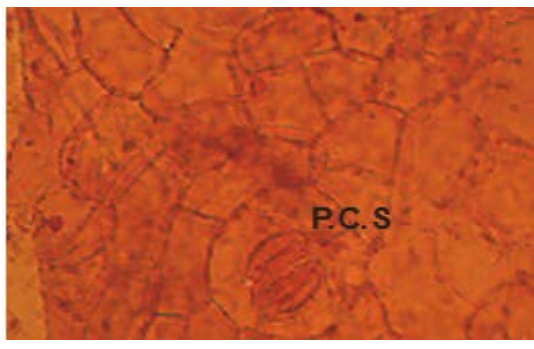

Plate 4T. P.C.S, Parallel contiguous stomata of $H$. rosa-sinensis (Upper Surface) $\mathrm{x} 400$

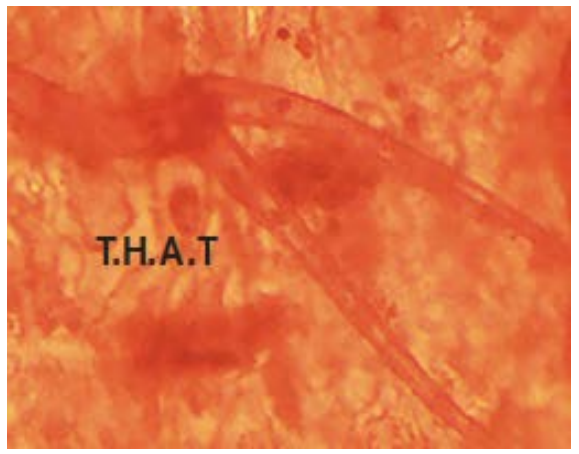

Plate 5A. T.H.A.T, Three-armed sessile stellate trichome of $H$. rosasinensis (lower Surface) x 400

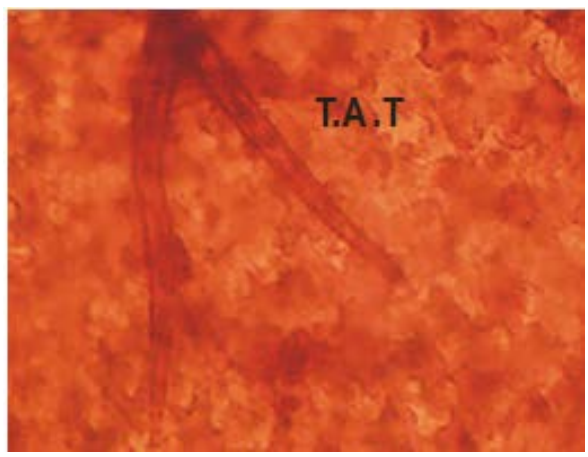

Plate 5B. T.A.T, Two-armed trichome (Bifurcated) of H. rosa-sinensis (lower Surface) x 400

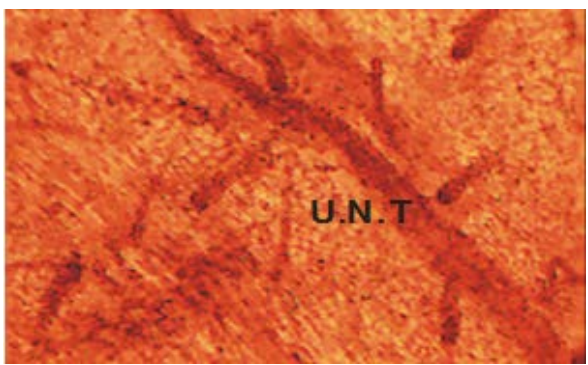

Plate 5C. U.N.T, Unicellular trichome of $H$. rosa-sinensis (lower Surface) x 100

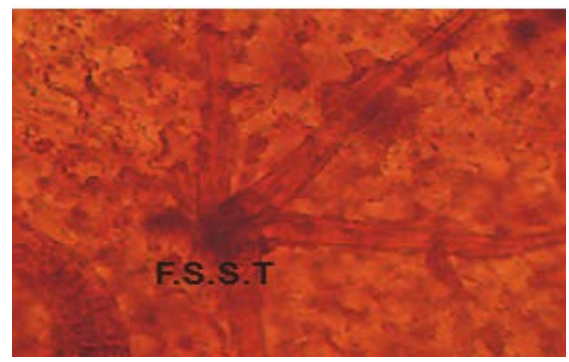

Plate 5D. F.S.S.T, Four-armed sessile stellate trichome of $H$. rosasinensis (lower Surface) x 400

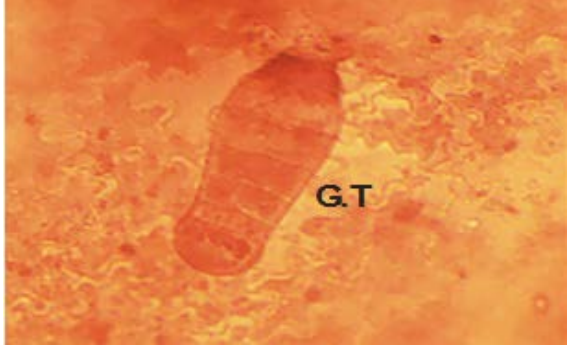

Plate 5E. G.T, Glandular trichome of H. rosa-sinensis (lower Surface) x 400

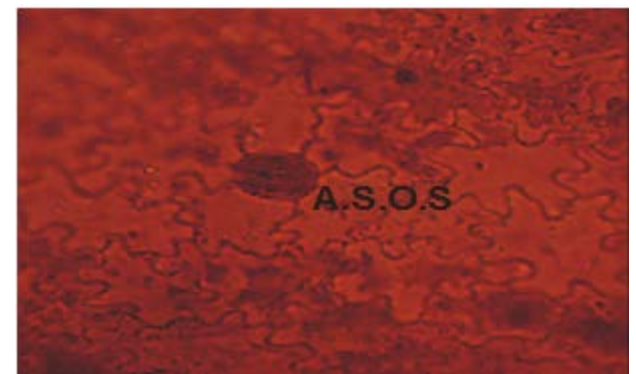

Plate 5F. A.S.O.S, Anomocytic stomata of $H$. rosa-sinensis (lower Surface) $\mathrm{x} 400$

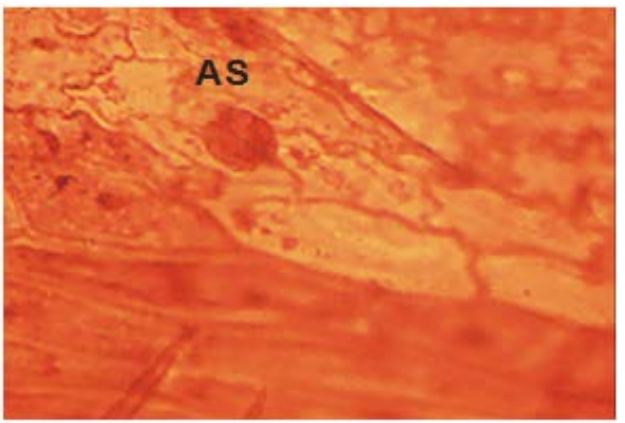

Plate 5G. A.S, Anomocytic stomata of H. rosa-sinensis (lower Surface) $\mathrm{x} 400$

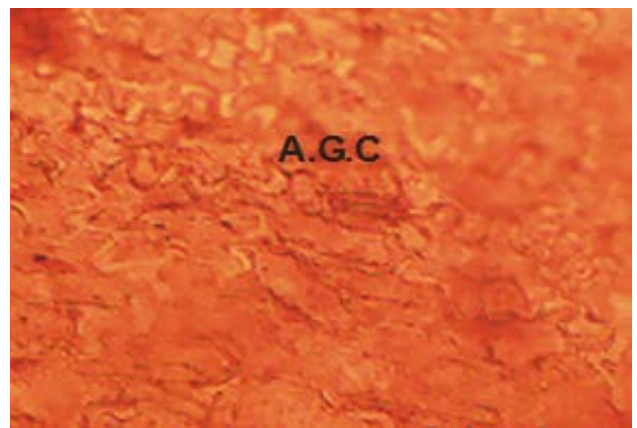

Plate 5H. A.G.C, Aborted guard cell of $H$. rosa-sinensis (lower Surface) $\mathrm{x} 400$

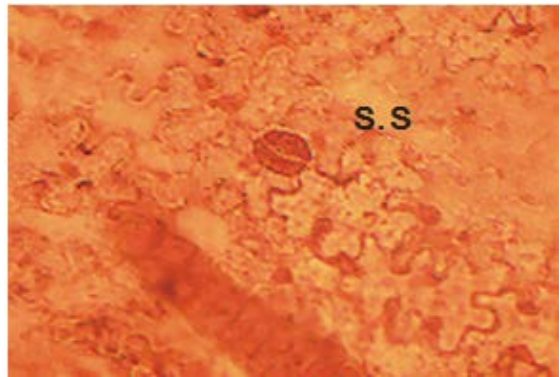

Plate 5I. S.S, Staurocytic stomata of $H$. rosa-sinensis (lower Surface) x 400 


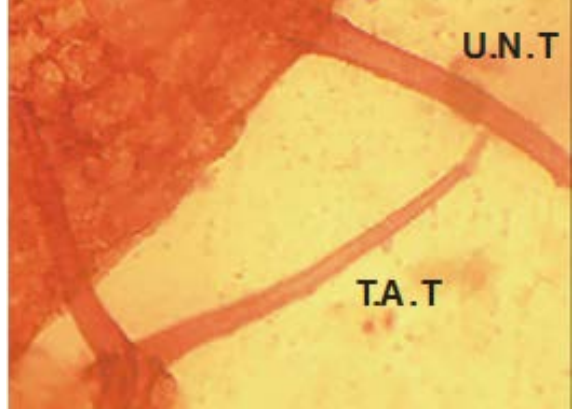

Plate 5J. T.A.T.T, Two-armed trichome (Bifurcated) and unicellular trichome of $H$. rosa-sinensis (lower Surface) x 400

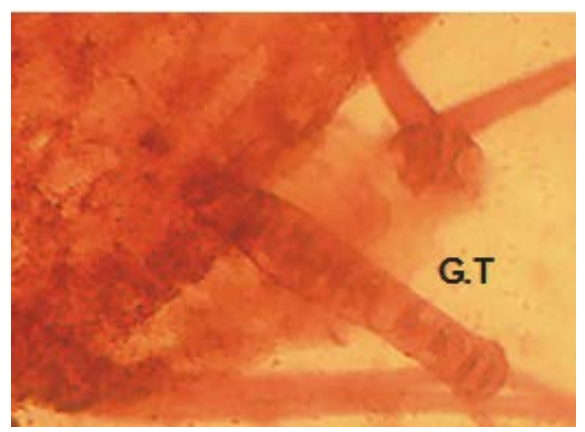

Plate 5K. G.T, Glandular trichome of H. rosa-sinensis (Upper Surface) x 400

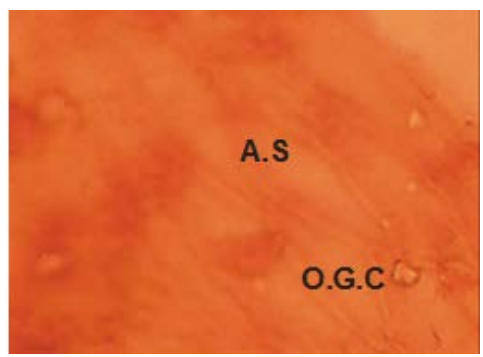

Plate 5L. A.S, Anisocytic stomata and O.G.C, one guard cell of H. rosasinensis (Upper Surface) x 400

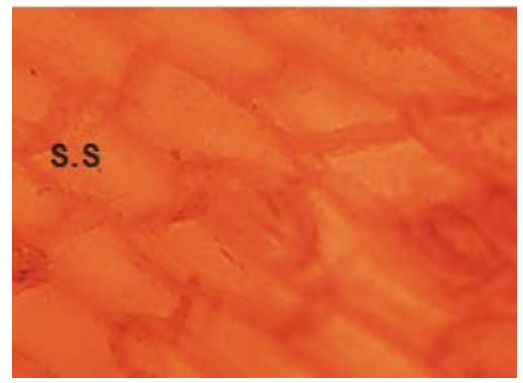

Plate 5M. S.S, Staurocytic stomata of $H$. rosa-sinensis (Upper Surface) x 400

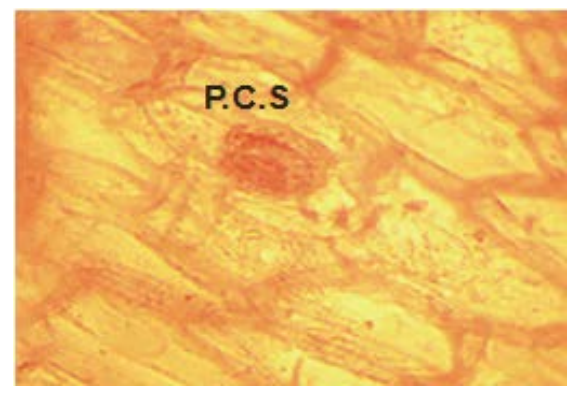

Plate 5N. P.C.S, Parallel contiguous stomata of $H$. rosa-sinensis (Upper Surface) $\mathrm{x} 400$

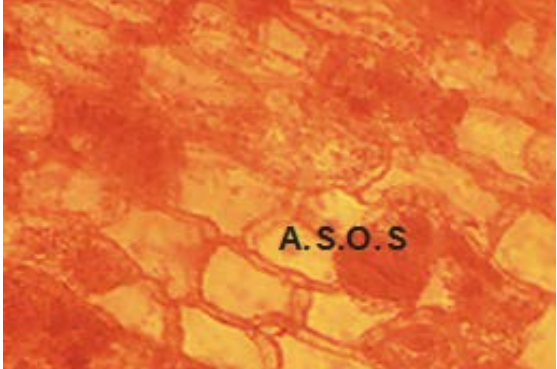

Plate 50. A.S.O.S, Anomocytic stomata of $H$. rosa-sinensis (Upper Surface) $\times 400$

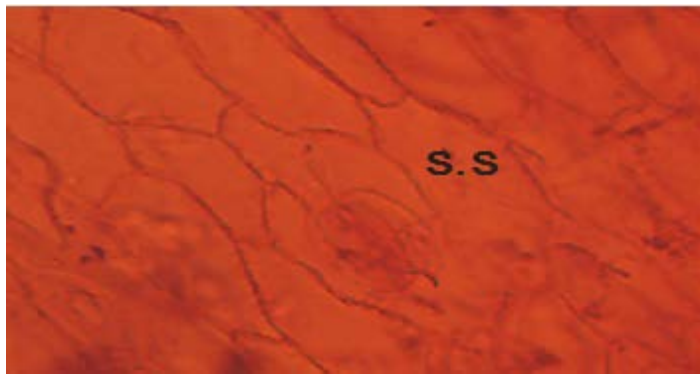

Plate 5P. S.S, Staurocytic stomata of $H$. rosa-sinensis (Upper Surface) $\mathrm{x}$ 400

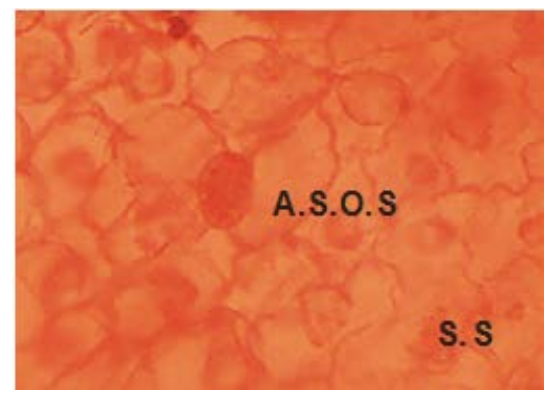

Plate 6A. A.S.O.S, Anomocytic and S.S, Staurocytic stomata of $H$. acetosella (Lower Surface) x 400

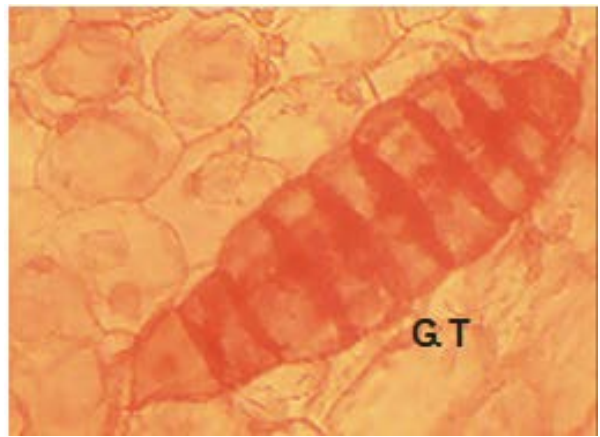

Plate 6B. G.T, Glandular trichome of $H$. acetosella (Lower Surface) x 400

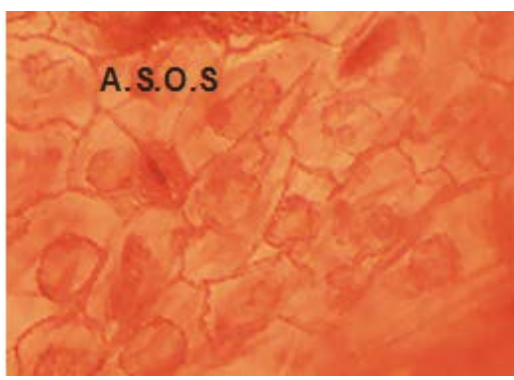

Plate 6C. A.S.O.S, Anomoxytic stomata of H. acetosella (Lower Surface) x 400 


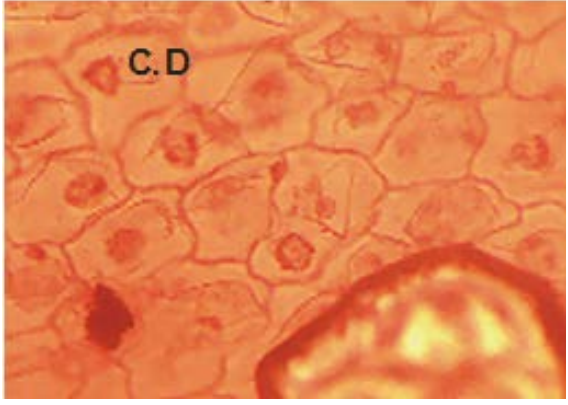

Plate 6D. C.D, Crystal druses of H. acetosella (Lower Surface) x 400

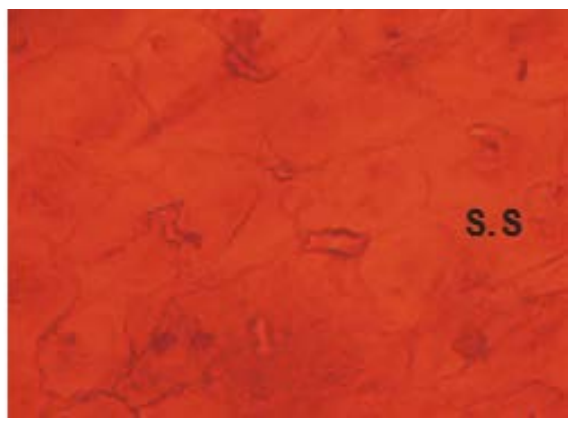

Plate 6E. S.S, Staurocytic ad aborted stomata of H. acetosella (Lower Surface) x 400

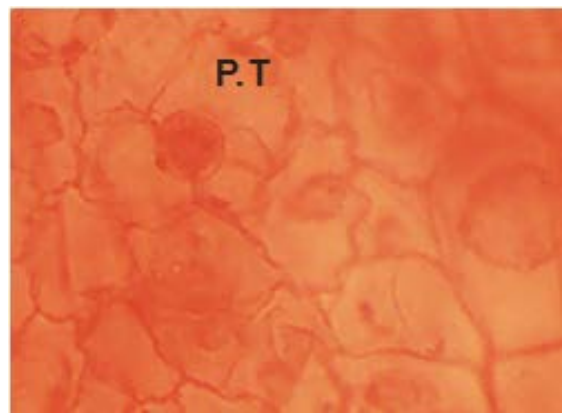

Plate 6F. P.T, Peltate trichome of $H$. acetosella (Lower Surface) x 400

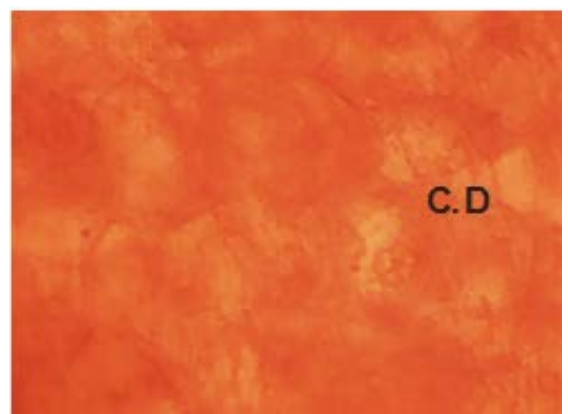

Plate 6G. C.D, Crystal druses of H. acetosella (Upper Surface) x 400

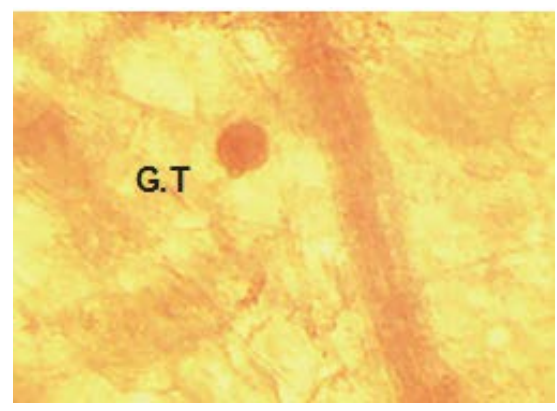

Plate 6H. G.T, Glandular trichome of H. acetosella (Upper Surface) x 400

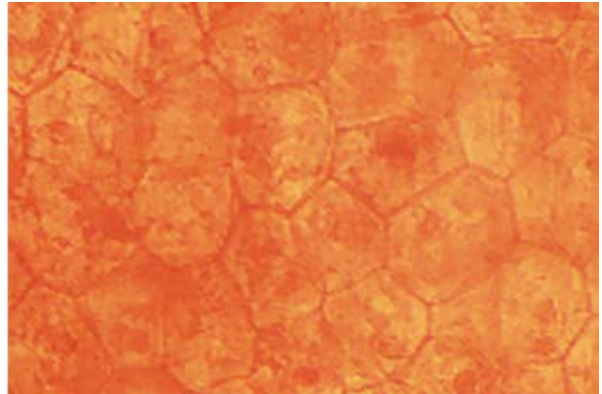

Plate 6I. E.P, Epidermal cell of H. acetosella (Upper Surface) x 400

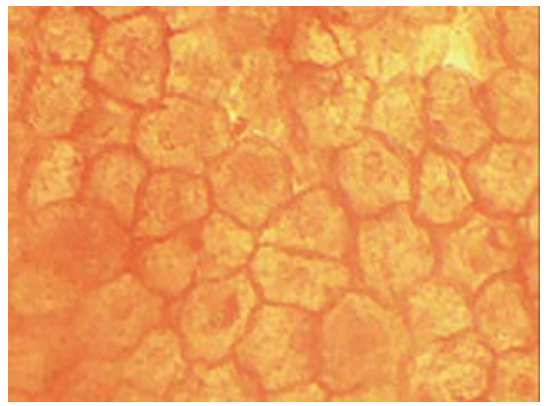

Plate 7A. E.P, Epidermal cell of H. acetosella (Upper Surface) x 400

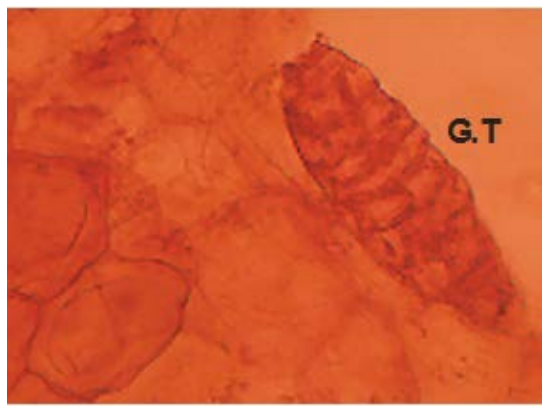

Plate 7B. G.T, Glandular trichome of $H$. acetosella (Lower Surface) x 400

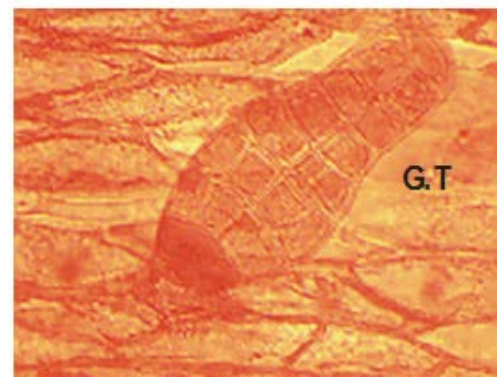

Plate 8A. G.T, Glandular trichome of $H$. arnottianus (Upper Surface) x 400

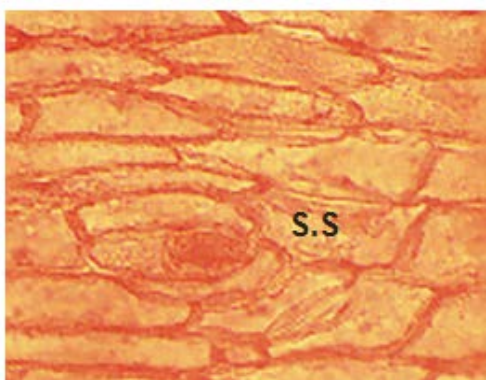

Plate 8B. S.S, Staurocytic stomata of $H$. arnottianus (Upper Surface) x 400 


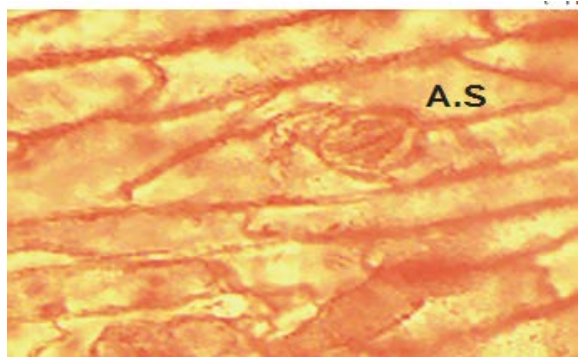

Plate 8C. E.P, Epidermal cell and A.S, Anisocytic stomata of $H$. arnottianus (Upper Surface) x 400

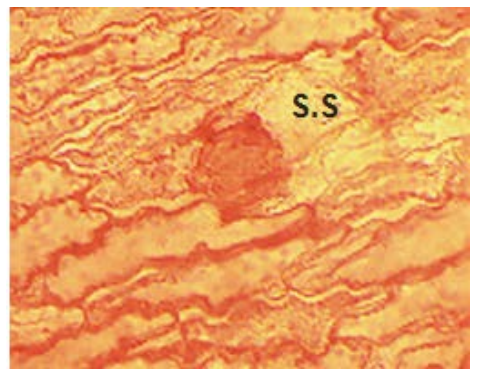

Plate 8D. S.S, Staurocytic stomata of H. arnottianus (Lower Surface) x 400

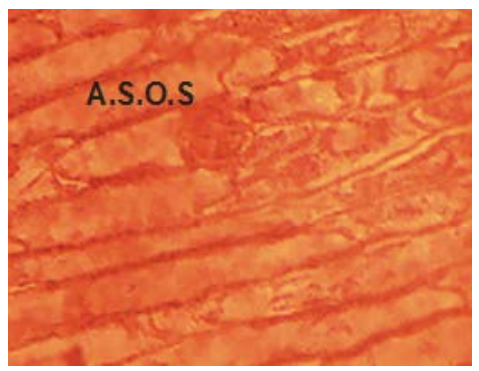

Plate 8E. A.S.O.S, Anomocytic stomata of $H$. arnottianus (Lower Surface) $\mathrm{x} 400$

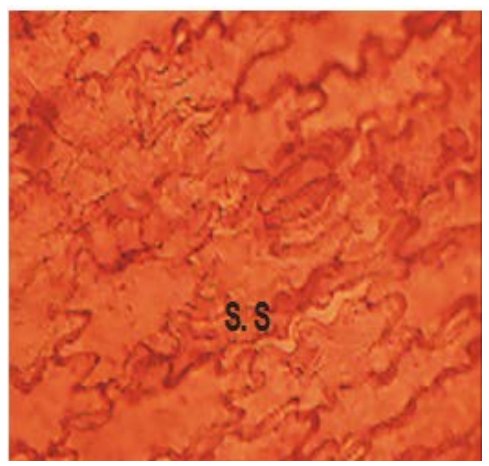

Plate 8F. S.S, Staurocytic stomata of H. arnottianus (Lower Surface) x 400

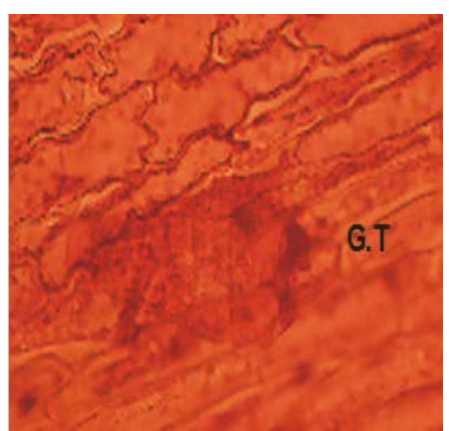

Plate 8G. G.T, Glandular trichome of $H$. arnottianus (Lower Surface) x 400

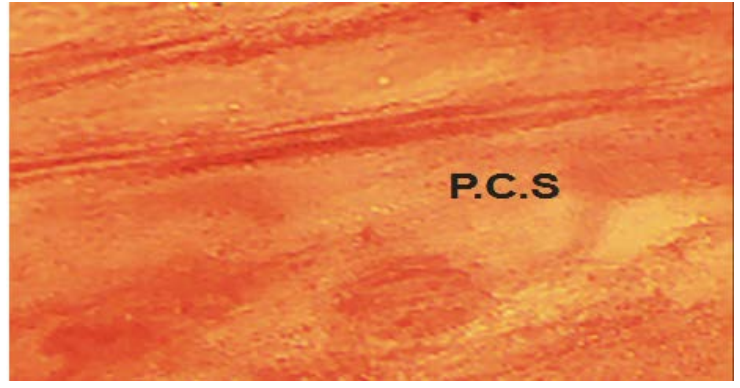

Plate 8H. P.C.S, Parallal contiguous of $H$. arnottianus (Lower Surface) x 400

\section{Discussion}

Anatomical features are widely used in systematic for identification, for placing anomalous group in satisfactory position in classification and for indicating patterns of relationship that may have been obscured by superficial convergence in anatomical features and morphology [29].

Anatomical evidence have proved useful in characterization of $H$. surattensis, $H$. arnottianus, $H$. acetosella and $H$. rosa-sinensis of the leaves and flowers respectively. Epidermis was pentangular to polygonal to irregular in shape and size with slightly undulating, undulate and sinous anticlinal walls. In $H$. surattensis, stomatas are absent on abaxial and adaxial surfaces of flower but present in both surfaces of the leaves (Hypostomatic) and mostly anisocytic with some brachyparacytic and staurocytic types. In H. arnottianus, stomatas are present on both surfaces of the leaves and flowers but scanty on the abaxial surface then abundance on adaxial surface (Hypoamphistomatic). On the species of Talinum, Essiett and Akpabio [30] shows that, it is possible for most species to have more than one type of stomata. The present of various types of stomata in the taxa is of taxonomic interest in this study, because it can distinguish $H$. acetosella as having laterocytic stomata on both surfaces of the leaves and present on the adaxial surface of $H$. surattensis only, while staurocytic stomata is abundantly present on the abaxial surface of $H$. rosasinensis flower but in addition, brachyparacytic stomata occurred on $H$. arnottianus leaves, both adaxial and abaxial surfaces but in flower, brachyparacytic are absent in all surfaces.

Another interesting character is the presence of abnormal stomata with one guard cell, an aborted guard cell, parallel contiguous stomata are present on abaxial surface of $H$. acetosella and adaxial surface of $H$. rosasinensis, two stomatas sharing one subsidiary cell and guard cells respectively. There is no evidence to suggest that all the abnormalities are the result of environmental factors which were confirmed by Dehnel [31]. The presence and combination of different types of stomata on the epidermal surfaces highlighted by Metcalfe and Chalk [32] can be useful in the classification and delimitation of the taxa they studied. Some attempts have been made to use stomatal characters as an aid to classification. The diversity of the stomata is now reported in a number of plates and example in Phyla nodiflora, Convolvulaceae and Asteraceae [33]. The function of stomata is associated with various physiological processes and with survival of each individual plant [29]. It is expected that stomatal 
structure will be under strong and highly integrated genetic control and that modification of stomata during evolution will reflect both general relationship and evolutionary trend. The guard cell area, stomatal index provides value that would serve as parameters for comparison among taxa.

The stomatal index is independent of the environment size or portion of the leaf surface. Size of the intervening epidermal cells are reliable factors for identification [32]. Trichomes were present on vein cells. Glandular trichome on the adaxial surface of $H$. surattensis leaves, abaxial surface of $H$. acetosella and on both surfaces of $H$. rosasinensis, present on abaxial surface of $H$. surattensis and it was totally absent on the adaxial surface of $H$. surattensis flower and non-glandular trichome on both surface of $H$. arnottianus and $H$. surattensis leaves but absent on both surfaces of the flower. Peltate trichomes are found on both surfaces of $H$. arnottianus. The high density of the long unicellular hairs in them also serve to reduce the rate of transpiration in the plants. The importance of trichomes in taxonomy has been highlighted by Stace [34] in the family Combretaceae, Illoh and Inyang [35].

Stomatal index varied between the four species of Hibiscus leaves on both adaxial and abaxial surfaces. The highest stomatal index was found on the leaf of $H$. rosasinensis on the adaxial surface (44\%). It is useful for the separation of $H$. surattensis flower (Table 3) in line with the findings of Isawumi [36] and Abdulrahaman and Oladele [37]. Stomatal index is independent on the environmental size of the leaf surface of the epidermal cell and it is a reliable factor for identification. The function of stomata was associated with various physiological process and success of each individual plant (Essiett, 2004). The guard cell area and stomatal index provides values that would serve as parameters for comparison among taxa. The importance of crystals in taxonomy as diagnostic tools was emphasized by Amos [38], he went further to mention that their mode of distribution is equally important in taxonomy. The presence of different types of crystals of calcium carbonate known as druses of crystals and their distribution in the leaves and flowers are therefore of interest. This was conflicted with Dehnel [31].

\section{Conclusion}

In conclusion, the analysis of these research works of $H$. arnottianus, $H$. surattensis, $H$. rosa-sinensis and $H$. acetosella have been distinguished on the basis of microscopy evaluation. The presence of different types of stomatas on the leaves and flowers of Hibiscus species studied shows that these bases provide some justification for the description, identification and collection of plant species.

\section{References}

[1] Stace, C.A, Plant taxonomy and Biosystematic. Comtemporary Biology, First Edition, John Willey and Sons Ltd., New York, 1980, 289.

[2] Wilson, F.D, A distributional and cytological survey of the presently recognized taxa of Hibiscus section Furcaria (Malvaceae). Bonplandia, 15 (1-2): 53-62, 2006.
[3] Kim, K.Y, Hibiscus. In: J. L. Longe: The Gale Encydopedia of alternative Medicine Farmington, Hills, Ml; Thomson/Gale, 2005, 200-205.

[4] Burkill, H.M, The useful plants of West Tropical Africa. $\left(2^{\text {nd }}\right.$ Edition): Royal Botanic Gardens Kew. 1995, 654-670.

[5] Sharma, S.N. and Sultana, S, Effect of Onosma echioides on DMBA/croton oil medicated carcinogenic response, hyperproliferation and oxidative damage in marine skin. European Journal of Cancer Prevention, 13: 40-53, 2004.

[6] Abdel-Monem, M.A., Zeinab, I.E. and Mona, F, Pharmacognostical study of Hibiscus trionum and Hibiscus rosasinensis, family Malvaceae, M.Sc. Thesis. Pharmacognosy Department, Faculty of Pharmacy, Zagzig University, Egypt, 2011, 102-108.

[7] Dafallah, A.A. and Al-Mustafe, Z, Investigation of the antinflamatory activity of Acacia gilotica and Hibicus sabdariffa. The American Journal of Chinese Medicine, 24: 263-269, 1996.

[8] Schdewa, G. and Khemani, T.H, Effects of some medicinal plant extract on anti-inflammatory and anti-pyretic using albino Wistar rats. Enthnopharm., 6. 78-84, 2003.

[9] Pale, E., Kouda, B.M. and Nacro, M, Characterization and antioxidative scavenging activities of anthocyanins in plants of Burkina Faso, Comptes Rendus Chimie, 7 (10-11). 973: 980, 2004.

[10] Hui, H., Jing, H.C., Wu, H.K. and Chau, J.W, Chempreventive properties of Hibiscus sabdariffa L on human gastric carcinoma cells through apoptosis induction and JNK/p38 MAPK signaling activation, Chemic-Biological Interactions, 165 (1). 59-75, 2007.

[11] Deyanira, O., Enrique, J.F., Alejandro, Z., Armando, H.A., Jaime, T. and Laura, A, Inhibition of Angiotensin Converting Enzyme (ACE) activity by the anthocyannins delphinidin-and cyaniding-30-sambubiosides from Hibiscus sabdariffa, Journal of Ethnopharmacology, 127.7-10, 2010.

[12] Chang, Y.C., Havang, H.P., Hsu, J.D., Yang, F. and Wang, J.C, Hibiscus antholyanins rich extract-induces apoptotic cell death in human promyelocytic leukemia cells, Toxicology and Applied Pharmacology, 205 (3). 201-2012, 2005.

[13] Mishra, M., Shukla, Y.N., Jain, S.P. and Kumar, S, Chemistry and Pharmacology of some Hibiscus species, J. Med Aromat. Plant Sci., 21 (4). 1169-1186, 1999.

[14] Tzu, L.L., Hui, H.L., Chang, C., Ming, C.L., Ming, C. and Chau, J.W, Hibiscus sabdariffa extract reduce serum cholesterol in men and women. Nutrition Research, 27 (3). 140-145, 2007.

[15] Gilani, A.H., Bashir, S., Janbaz, K.H. and Shah, A.Z, Presence of cholinergic and calcium channel blocking activities explains the traditional use of Hibiscus rosa-sinensis in constipation and diarrhoea, Journal Ethnopharmacology, 102.94-289, 2005.

[16] Levin, D.A, The role of trichiomes in plant defense. Q. Rev-Bio., 48.3-15, 1993.

[17] Marquis, R.J, The selective impact of herbivory In: Fritz, R.S. and Simms, E.L, (eds.), Plant Resistance to Herbivores and Pathogens, Ecology, Evolution and Genetics, University of Chicago Press, Chicago, 1992, 301-325.

[18] Hare, J.D. and Smith, J.L, Competition, herbivory and reproduction of trichomes phenotypes of Datura Wright II. Ecd., 86,334-339, 2005.

[19] Ehleringe, J, Ecology and ecophysiology of leaf rebescence in North American desert plants. In: Rodregues E, Healey PL and Mehita I (Eds), Biology and chemistry of plant trichomes, Plenum Press, New York, 1984, 114-132.

[20] Larcher, W, Physiological Plant Ecology. Springerverlag, Berlin, 2001, 100-109.

[21] Yan-Ming, F. and Ru-Wen, F, Variation and Evolution of Leaf trichomes in Chinese Hamamelidaceae. Acta. Phytotaxon. Sin., 31.147-152, 1993.

[22] Spring, O, Chemotaxonomy based on Metabolites from Glandular Trichomes. Adv. Bot. Res., 31. 152-174, 2000.

[23] Rao, S.R.S. and Ramayya, N, Structure Distribution and Taxonomic Importance of Trichomes in the Indian Species of Malvastrum. Phytomorphola; 24. 40-44, 1977.

[24] Adedeji, O. and Illoh, H.C, Comparative foliar anatomy of ten species in the genus Hibiscus Linn. In Nigeria. New Bot., 31.147180, 2004.

[25] Nwachuhwu, L. and Mbagwu, G, Anatomical features of the roots and leaves of Hibiscus rosa-sinensis and Abelmoschus esculenta. Plant Sci., 3. 224-227, 2007.

[26] Priya, V.D., Ninan, C.A. and Kothari, N.M, Stomatal variations in cultivars of Hibiscus rosa-sinensis L. Biodiversity Conservation, 9.129-134, 2008. 
[27] Franceschi, V.R. and Horner, H.T, Calcium Oxalate crystals in plants. Bot. Rev., 46. 361-427, 1980.

[28] Wang, Z.Y., Gould, K.S. and Patterson, K.J, Structure and development of Mucilage-crystal Idioblasts in the roots of five Actinidia species. Int. J. Plant Sci., 155. 342-349, 1994.

[29] Essiett, U.A, Biosystematic studies of some Nigerian Dioscorea species. Unpublished Ph. D. Thesis, Department of Botany and Microbiology, University of Uyo, Uyo, Nigeria. 2004.

[30] Essiett, U.A. and Akpabio, K.E, The Comparative Anatomy of Talinum triangulare and Talinum portulacifolium in Nigeria. Academic and Scientific Research Institute Publication. Int. J. Biotechnol and Allied Sci, 4 (1). 424-432, 2009.

[31] Dehnel, G.S, Response of Stomata to Wounding. Botanical Gazette, 122. 124-130, 1960.

[32] Metcalfe, C.R. and Chalk, L, Anatomy of Dicotyledons, $2^{\text {nd }}$ Edition. Vol.1, Oxford University Press, London, 1979, 276.
[33] Akpabio, K.E., Essiett, U.A. and Nwokafor, B.N, The taxanomic significance of certain anatomical variation among the Asteraceae. Nigerian Journal of Botany, 20 (1). 149-155, 2007.

[34] Stace, C.A, Plant taxonomy and Biosystematic. Comtemporary Biology, First Edition, John Willey and Sons Ltd., New York, 1980, 289.

[35] Illoh, H.C. and Inyang, U.E, Foliar Epidermis and Petiole Anatomy in some Nigeria Solanum Linn. Species in the sub-genus Leptostemonum (Bitt). Dun. Glimpses in Plant Research, 12. 7386, 1998.

[36] Isawumi, M.A, Epidermal studies in the species of Jatropha L. (Euphorbiaceae) found in Nigeria. Nigeria Journal of Botany, 23. 94-100, 1986.

[37] Abdulrahaman, A.A. and Oladele, F.A, Stomatal size, Density and index in some vegetable species in Nigeria. Nigeria Journal of Botany, 16. 144-150, 2003.

[38] Amos, G.I, Some Siliceous timbers of Bitter Guyana. Caribb. For., 12. 133-137, 1951. 